\title{
Article \\ Performance of Optical Structural Vibration Monitoring Systems in Experimental Modal Analysis
}

\author{
Maksat Kalybek ${ }^{1, *}$, Mateusz Bocian ${ }^{1,2}$ (D) and Nikolaos Nikitas ${ }^{3}$ (D) \\ 1 School of Engineering, University of Leicester, Leicester LE1 7RH, UK; m.bocian@leicester.ac.uk \\ 2 Dynamics, Vibration \& Acoustics Laboratory, University of Leicester, Leicester LE1 7RH, UK \\ 3 School of Civil Engineering, University of Leeds, Leeds LS2 9JT, UK; n.nikitas@leeds.ac.uk \\ * Correspondence: mk595@leicester.ac.uk
}

Citation: Kalybek, M.; Bocian, M.; Nikitas, N. Performance of Optical Structural Vibration Monitoring Systems in Experimental Modal Analysis. Sensors 2021, 21, 1239. https://doi.org/10.3390/

s21041239

Academic Editor: Paulo Antunes

Received: 4 January 2021

Accepted: 8 February 2021

Published: 10 February 2021

Publisher's Note: MDPI stays neutral with regard to jurisdictional claims in published maps and institutional affiliations.

Copyright: (c) 2021 by the authors. Licensee MDPI, Basel, Switzerland. This article is an open access article distributed under the terms and conditions of the Creative Commons Attribution (CC BY) license (https:/ / creativecommons.org/licenses/by/ $4.0 /)$.

\begin{abstract}
Image-based optical vibration measurement is an attractive alternative to the conventional measurement of structural dynamics predominantly relying on accelerometry. Although various optical vibration monitoring systems are now readily available, their performance is currently not well defined, especially in the context of experimental modal analysis. To this end, this study provides some of the first evidence of the capability of optical vibration monitoring systems in modal identification using input-output measurements. A comparative study is conducted on a scaled model of a 3D building frame set in a laboratory environment. The dynamic response of the model to an impulse excitation from an instrumented hammer, and an initial displacement, is measured by means of five optical motion capture systems. These include commercial and open-source systems based on laser Doppler velocimetry, fiducial markers and marker-less pattern recognition. The performance of these systems is analysed against the data obtained with a set of high-precision accelerometers. It is shown that the modal parameters identified from each system are not always equivalent, and that each system has limitations inherent to its design. Informed by these findings, a guidance for the deployment of the considered optical motion capture systems is given, aiding in their choice and implementation for structural vibration monitoring.
\end{abstract}

Keywords: optical systems; structural health monitoring; modal testing; vibration measurement; experimental modal analysis

\section{Introduction}

Optical motion capture systems (MCS) are becoming increasingly popular in structural vibration monitoring [1-3]. Their main advantage lies in the remote operation hence avoidance of the deployment of cabling system associated with conventional motion capture systems, most often relying on accelerometry. Although optical MCS derive the most accurate results tracking a single point only, continuous improvement in resolution of all sorts of digital cameras has enabled vibration tests to be performed in which multiple points are being tracked simultaneously. For example, Park et al. [4] presented results from tests utilising motion capture system (MCS) based on three cameras measuring 3D structural displacements in a laboratory environment. The process required installing multiple light-reflective markers on the structure, each seen by at least two cameras at any time. A number of studies explored the capabilities of single-camera systems. For example, Brownjohn et al. [5] and Luo et al. [6] used a single high speed camera coupled with custom software for tracking multiple points on bridges in situ. Patil et al. [7] proposed a method of stitching together mode shapes from data obtained from video footage of a pair of cameras roving around a structure for 3D measurements. As for the equipment, all these studies used off-the-shelf commercial products dedicated to motion capture.

Optical MCS could become a cost-effective solution for structural vibration monitoring, particularly when direct displacement rather than acceleration is sought. However, off-theshelf commercial products from the leading suppliers carry a cost of tens of thousands GBP, 
which may be prohibitive for many potential users. Undoubtedly, these solutions are quite refined in terms of data processing capabilities and user-friendly interfaces. However, it remains to be determined whether simpler solutions relying on consumer-grade hardware and well documented data processing algorithms, often available in open-source format, can match their performance, or at least offer enough measurement fidelity to be considered viable alternative solutions. The results of recent investigations are encouraging in this respect. For example, Kromanis et al. [8] compared a number of modern smartphones against various cameras by coupling them with various image processing algorithms. By measuring deformations of structural elements caused by static, quasi-static and dynamic loads in the lab, they demonstrated the feasibility of such low-cost systems for structural health monitoring (SHM). A number of studies explored the capabilities of smartphone cameras in capturing the vibration and displacement of structures in situ $[9,10]$, whilst other studies used action cameras for the same purpose. For example, Lydon et al. [11] explored a feature-based template matching technique [12] by tracking the displacement of a single point on two structures using Go-Pro camera. Xu et al. [13] conducted a similar study, but using area-based template matching technique [12] for tracking multiple points. A few attempts were also made to use other types of optical MCS, such as depth sensing cameras (RGB-D) [14] and video cameras [15,16], while an exhaustive review on relevant advances can be found elsewhere [3,17]. However, none of the studies provided a full dynamic characterisation of the tested structures, which is the ultimate goal of modal analysis [18].

A continuing effort is being made on developing advanced modal identification techniques based on operational modal analysis (OMA), in which the excitation force comes from either environmental and/or man-made sources, see e.g., the hybrid OMAX [19], associated with the operational regime of the investigated structure [20]. However, while pure OMA can provide information about the modal frequencies, damping, and mode shapes, it cannot intrinsically scale the mode shapes since it relies on response measurement only while making rather specific assumptions as to the nature of the excitation input [21]. Although a number of approaches has been proposed in the last two decades to overcome this limitation [22], their application requires either various types of interventions on the structure or its numerical model [23]. The former approach is realised by introducing a local change in the mass or stiffness of the structure, or an auxiliary mechanical system. Therefore, it carries a similarity with experimental modal analysis (EMA) in which an extraneous device is employed-here a force source, enabling direct scaling of the mode shapes with modal mass. The latter approach relies on a highly reliable finite element model, which is notoriously difficult to obtain even for seemingly simple civil structures. Consequently, EMA-based practices are still the gold standard in full dynamic characterisation of structures.

Although studies on full-scale structures in situ are the ultimate reason for interest in optical MCS within civil engineering community, laboratory-based experiments allow the MCS performance to be examined in a controlled environment while excluding any environmental effects which can debilitate the measurement accuracy. Such studies generally involve using simple structural elements, most often beams or columns [16], or custom-built downscaled models of simple structures, such as few-storeys high models of building frames [4,24-27]. The latter approach, with a more demanding variant of a 3D rather than a planar frame, is adopted in this study.

To the best of the authors' knowledge, this is the first study aiming to assess the performance of various optical structural vibration monitoring systems in the context of EMA. Six instrumentation systems, including five optical MCS, are deployed in a laboratory environment to measure the response of a scaled frame of a 3D building to the impulse excitation from an instrumented hammer and initial displacement. A complete set of modal properties is used for benchmarking, including natural frequencies, damping ratios, mode shapes and modal (generalised) masses. The damping ratios and in particular modal masses seem to have never appeared in alike comparisons involving image-based optical MCS. 
The rest of the paper is organised as follows. Section 2 presents the tested structure, briefly introduces instrumentation systems used in this study, describes experimental protocols and outlines data analysis procedures. The results are presented and discussed in Section 3. This includes the results of pose reconstruction assessment of ArUco markers in Section 3.1, modal identification in Section 3.2 and initial displacement response in Section 3.3. The conclusions are presented in Section 4.

\section{Methodology}

\subsection{Tested Structure}

A simple steel frame shown in Figure 1 was designed and built at the University of Leicester, UK. SAP2000 version 20 structural analysis package was used in the specification of structural components such as to tune natural frequencies of the frame to those representative of full-scale civil structures such as short-span bridges and medium-rise buildings. The frame has six plates above the bottom plate centrally bolted onto the floor, each $20 \mathrm{~mm}$ thick and cut to fit within $180 \times 180 \mathrm{~mm}$ outline, joined by four columns recessed into the plates' corners. Mild steel with density $7850 \mathrm{~kg} \cdot \mathrm{m}^{-3}$ was used throughout. The distance between centroids of each two consecutive plates is $200 \mathrm{~mm}$, except for the distance between plate 4 and 5 counting from the bottom, which is $180 \mathrm{~mm}$. The columns have rectangular cross section of $10 \times 3 \mathrm{~mm}$ and are connected to each plate using two screws arranged one above the other. The structure is rather lively, i.e., after providing a hit to one of the plates the structure can be seen with naked eye to vibrate for a prolonged time, indicating low damping. The structure together with its coordinate axes is shown in Figure 2. The movement in $z$-axis was of particular interest since it is associated with the weak (in terms of resistance of the section to bending/rotation) axis of the columns.

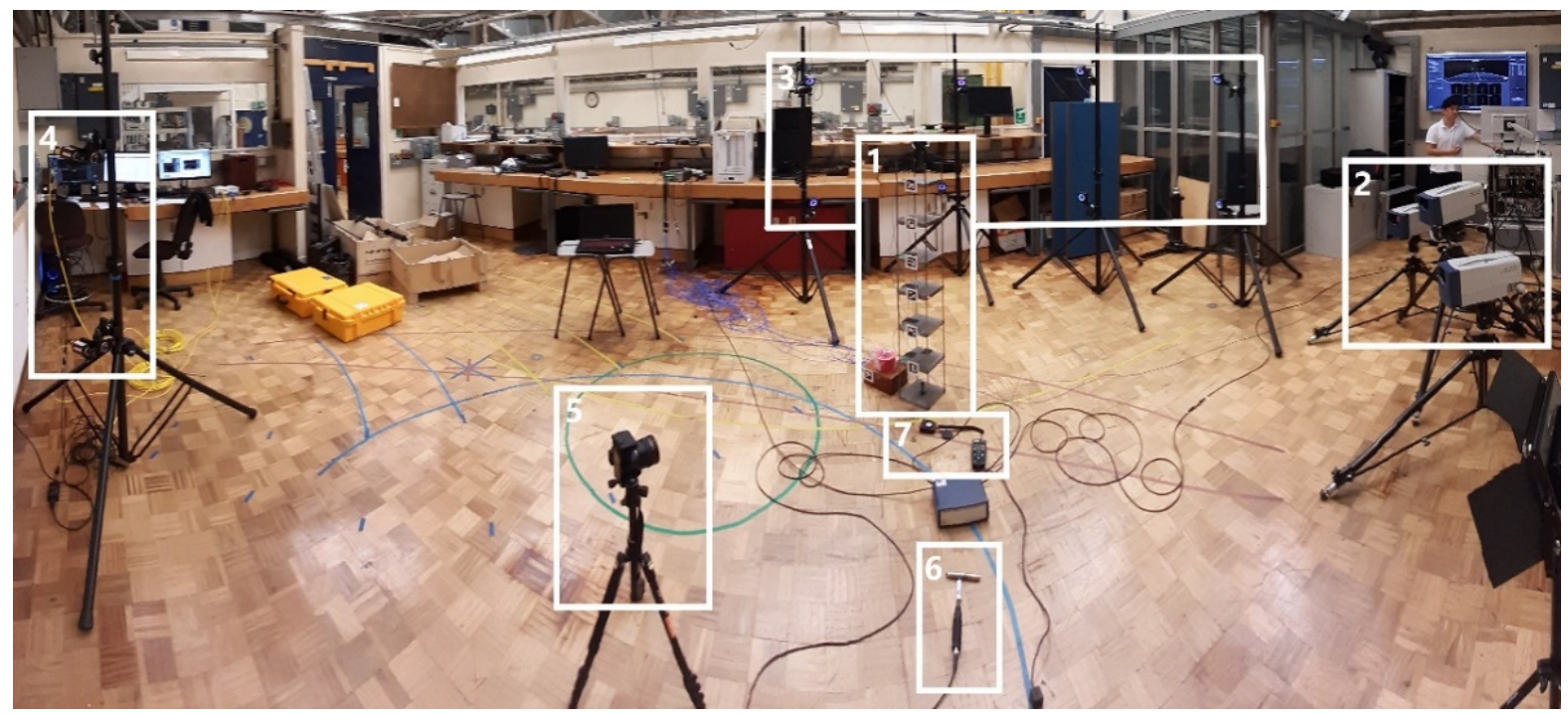

Figure 1. The layout of instrumentation systems in the Dynamics, Vibration, and Acoustics Laboratory at the University of Leicester: 1-Steel frame with accelerometers attached; 2-Polytec laser Doppler vibrometer; 3-OptiTrack; 4-Imetrum; 5-Consumer-grade camera (CGC); 6-instrumented hammer; 7-lux meter. 
(a)

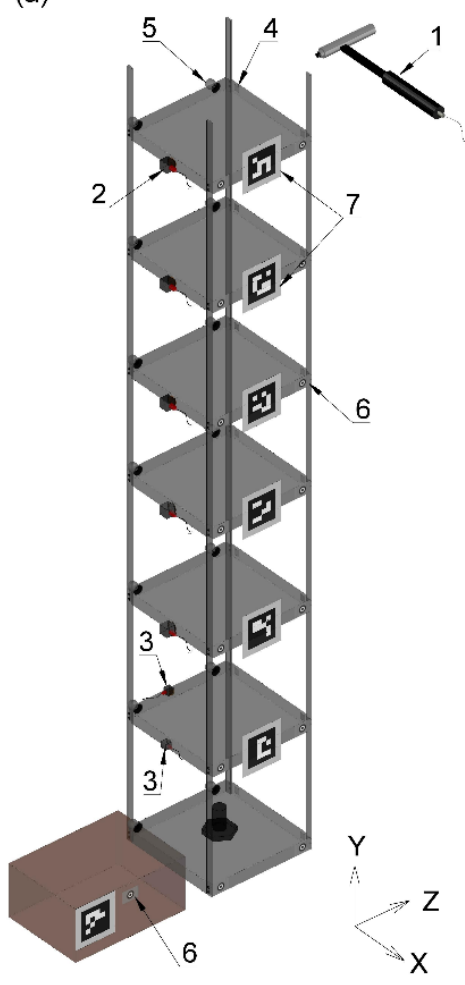

(b)

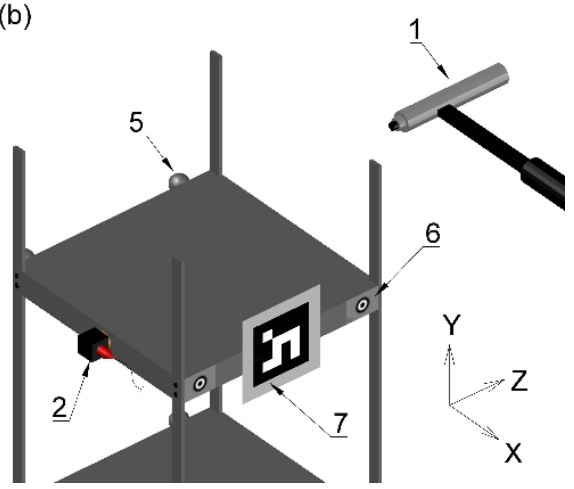

(c)

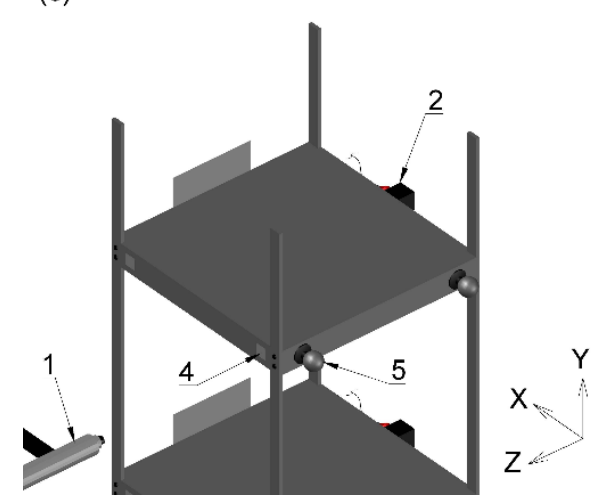

Figure 2. (a) Steel frame and $(\mathbf{b}, \mathbf{c})$ its details showing the positioning of sensors and markers around the top plate. 1-instrumented hammer; 2 -triaxial accelerometer; 3 - single axis accelerometer; 4-light reflective fiducial marker for laser Doppler vibrometer (LDV); 5 -spherical reflective marker for OptiTrack; 6-Imetrum marker, 7-ArUco marker.

\subsection{Instrumentation}

A set of conventional constant current accelerometers and five optical motion capture systems, including two systems relying on images recorded with a consumer-grade camera (CGC), were used in this study. The basic specification of these systems is given in Table 1. An instrumented hammer was used to provide force excitation and a lux meter was used to measure light intensity during the tests. A brief description of these instrumentation systems is given in Sections 2.2.1-2.2.7. Figure 2 presents the layout of accelerometers and fiducial markers attached to the frame, used in conjunction with optical MCS, and the point of application of excitation force with the instrumented hammer, which is the centroid of the side face of the top plate. This point was chosen with an intention not to mobilise strongly torsional modes which could distort the results of modal analysis from instrumentation systems which were not inherently capable of 3D measurements. It can be seen in Figure 2 that the accelerometers and markers cover all side faces of the plates above the bottom plate. The layout of the instrumentation and markers was optimised such as to obtain maximum quality measurements in z-axis. For clarity of presentation, the operational principles behind the different fiducial markers are explained in Section 2.3. Basic specification of the MCS used in this study is given in Table 1. 
Table 1. Basic specification of the motion capture systems used in this study.

\begin{tabular}{|c|c|c|c|c|c|}
\hline System & Sensor & Quantity & $\begin{array}{l}\text { Sampling } \\
\text { Frequency and } \\
\text { Resolution } \\
\end{array}$ & $\begin{array}{l}\text { Distance Relative } \\
\text { to the Markers }\end{array}$ & $\begin{array}{l}\text { Fiducial } \\
\text { Marker }\end{array}$ \\
\hline Polytec LDV & PSV-500-HM & 3 & $\begin{array}{c}200 \mathrm{~Hz} \text { and } \\
0.02 \mu \mathrm{m} / \mathrm{s}\end{array}$ & $3 \mathrm{~m}$ & \\
\hline OptiTrack & $\begin{array}{l}\text { Prime } 13 \text { with } \\
5.5 \mathrm{~mm} \text { lens }\end{array}$ & 8 & $120 \mathrm{fps}$ at $1.3 \mathrm{MP}$ & $1-3 \mathrm{~m}$ & \\
\hline Imetrum & $\begin{array}{l}\text { Manta G-223 B } \\
\text { NIR with } \\
16 \mathrm{~mm} \text { lens }\end{array}$ & 2 & $50 \mathrm{fps}$ at $2 \mathrm{MP}$ & $3 \mathrm{~m}$ & \\
\hline Accelerometers & $\begin{array}{l}\text { PCB } 356 \text { A16 } \\
\text { PCB } 333 \text { B30 }\end{array}$ & $\begin{array}{l}5 \\
2\end{array}$ & $\begin{array}{l}64 \mathrm{~Hz} \text { at } 100 \mathrm{mV} / \mathrm{g} \\
64 \mathrm{~Hz} \text { at } 100 \mathrm{mV} / \mathrm{g}\end{array}$ & $\begin{array}{l}\text { Direct contact } \\
\text { Direct contact }\end{array}$ & $\mathrm{N} / \mathrm{A}$ \\
\hline $\begin{array}{c}\text { CGC } \\
\text { (used with feature-based } \\
\text { ArUco and area-based } \\
\text { template matching) }\end{array}$ & $\begin{array}{l}\text { Cannon EOS } 200 \text { D } \\
\text { with DIGIC } \\
7 \text { processor and } \\
20 \text { mm Canon lens }\end{array}$ & 1 & $\begin{array}{c}59.94 \mathrm{fps} \text { at } 24.2 \\
\text { MP }\end{array}$ & $1.5 \mathrm{~m}$ & \\
\hline
\end{tabular}

\subsubsection{Accelerometers}

Acceleration in $x$ - and $z$-axis (see Figure 2) was measured with five triaxial accelerometers (PCB 356 A16, USA) mounted onto plates 3-7, counting from the base (i.e., bottom) plate, and two single axis accelerometers (PCB 333 B30, USA) mounted onto plate 2 . The positions of accelerometers measuring the response in $z$-axis were collinear since they were located at the centroids of the plates' faces on the same side of the frame, as shown in Figure 2a.

\subsubsection{Imetrum}

Imetrum [28] is a commercial-grade MCS used for structural testing in a variety of applications in a laboratory and outdoor environment [5,29]. The main asset of the system is the Video Gauge software, developed based on the study of Macdonald et al. [30], which uses Digital Image Correlation (DIC) technique coupled with various prediction and subpixel refinement techniques. The setup used in this study consisted of a dedicated PC with Video Gauge software package, two synchronised cameras with 2 megapixel (MP) resolution equipped with $25 \mathrm{~mm}$ camera lenses, recording at 50 frames per second (fps) and mounted on a single tripod approximately $1.2 \mathrm{~m}$ apart. To perform 3D measurements, the calibration process includes specifying the inter camera distances and angles, and predefining some known distances within the captured image. 2D markers consisting of slightly blurred black and white concentric circles printed on white matte vinyl stickers were attached to the plates, as shown in Figure 2b, for improved tracking quality as the steel plate surface was found to be rather featureless at the scale of interest.

\subsubsection{OptiTrack}

OptiTrack [31] is a commercial-grade MCS used predominantly for laboratory testing in biomechanics, robotics and virtual reality applications. The setup used in this study consisted of eight Prime 13 cameras with 1.3 MP resolution and sampling at $120 \mathrm{fps}$, mounted in pairs on four tripods, a network hub and Motive 2.1.1 motion capture software. The system detects and tracks the movement of spherical reflective markers which were attached to the plates, as shown in Figure 2. A $250 \mathrm{~mm}$ T-shaped wand and $200 \mathrm{~mm}$ square were used to calibrate the cameras and define the coordinate system aligned with that of the frame. 


\subsubsection{Polytec Laser Doppler Vibrometer (LDV)}

Polytec laser Doppler vibrometer (LDV) PSV-500-3D was used in this study. It consisted of three HeNe red laser scanning heads enabling 3D measurement, one of which includes video camera facilitating system set up. The scanning heads were positioned at a distance of about $3 \mathrm{~m}$ from the frame and, since they utilise the Doppler effect, they were pointing at the plates' faces perpendicular to the $z$-axis at the angles close to 90 degrees to obtain reliable measurements of the structural response in $z$-axis. A proprietary PC with data acquisition board and PSV software was used during measurements. In order to perform 3D scan, all three laser heads were set up to measure one point of interest at a time. For better reflection of laser signals hence improved signal to noise ratio, 2D light reflective fiducial markers were attached to the plates of the structure as shown in Figure 2c.

\subsubsection{Consumer-Grade Camera (CGC)}

Canon EOS 200D CGC coupled with $20 \mathrm{~mm}$ focal length lens, with maximum aperture $\mathrm{f} / 2.8$, was used in this study, set to operate in a fixed focus mode. The video footage recorded with CGC was used with two MCS, (i) ArUco system tracking feature-based fiducial markers and (ii) area-based template matching system hereafter referred to as template matching and abbreviated by TM. The data processing was implemented in a custom application written in $\mathrm{C}++$ programming language, referring to open source OpenCV library of computer vision functions [32]. Sub-pixel refinement was used in both CGC-based MCS.

\section{Feature-Based Template Matching (ArUco)}

ArUco system, presented in Garrido-Jurado et al. [33], has so far been mainly used for pose estimation in computer vision applications such as robot navigation and augmented reality. It is believed to be one of the most evolved tools for fiducial marker detection [34]. The markers are composed of a wide black border and an inner binary matrix which determines their unique IDs. Marker tracking is performed in two stages: (i) Marker detection and (ii) estimation of markers' pose relative to the camera. During stage (i) the outer square box and the unique pattern of markers' inner binary code are identified. This inner pattern allows robust detection of multiple markers in an image frame. During stage (ii) the position and rotation of camera related to each marker is identified through a full projection matrix [35]. This is a camera calibration process where camera intrinsic parameters are identified using the images of a chessboard and then the camera extrinsic parameters are identified through the known physical geometry of the markers. For this study, the movement of the markers attached to the steel frame was identified relative to their state in the first frame of the captured video.

\section{Area-Based Template Matching}

The video from CGC used with ArUco was also used in an area-based template matching system, which in various forms is now being widely used for SHM [2,36]. The area-based template matching relies on the identification of template patterns within captured images. The normalised version of sum of squared differences (NSSD) [32] method was used as a correlation criterion. For improved computational efficiency, a region of interest (ROI) was defined, reducing the area of images within which to search for the template patterns. An offline calibration with chessboard was performed to remove camera distortion effects. A planar homography matrix was determined through linking the user-defined planar coordinates of key points on the 2D structural coordinate system and the pixel coordinates of these points. The area-based template matching system, hereafter simply referred to as template matching, used the ArUco markers to aid in pattern recognition. 


\subsubsection{Instrumented Hammer}

PCB 086C01 instrumented hammer was used to provide excitation energy to the structure. A pre-trigger offset was set based on the force threshold to ensure the whole signal was contained within the measured window. The hammer tip was chosen such as to ensure there is enough excitation energy within the whole frequency range of interest. An example of the recorded hammer force in time and frequency domains is shown in Figure $3 a, b$. The time and frequency ranges were truncated to highlight the shape of the force pulse and the magnitude of the FFT of the force within the frequency range of interest, respectively.

(a)

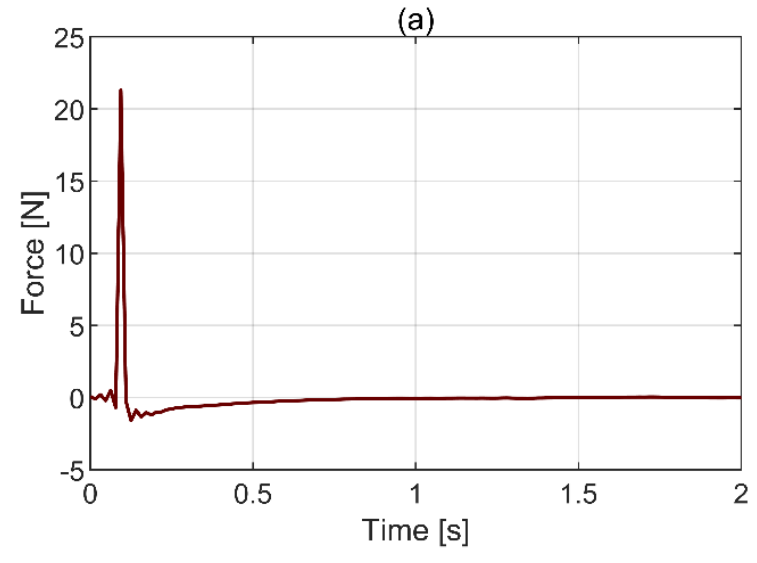

(b)

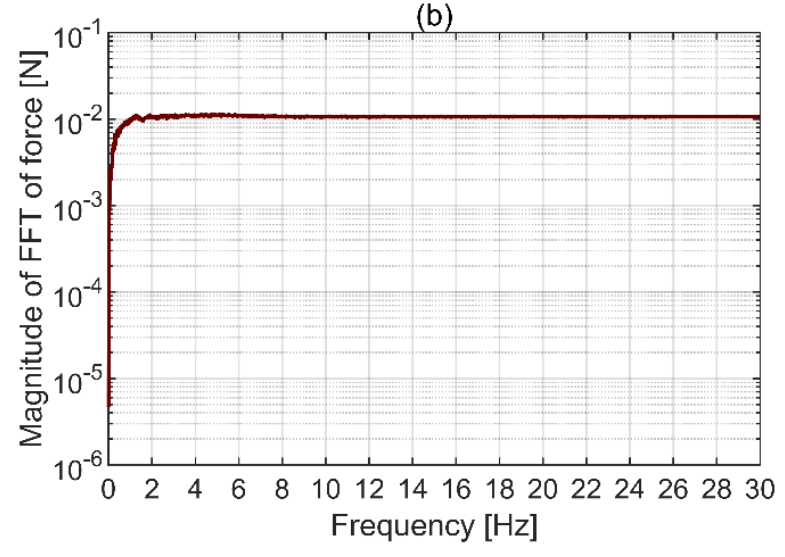

Figure 3. Hammer force in (a) time domain and (b) frequency domain.

The ripples on both sides of the pulse in Figure 3a are a consequence of applying anti-aliasing (low pass) filter on the force signal and indicate its frequency content goes beyond the limit set in data acquisition. This does not affect the quality of the analysis presented in this study [23]. It can be seen in Figure 3b that the force spectrum between $1.5 \mathrm{~Hz}$ and $30 \mathrm{~Hz}$ is flat hence there is energy in the frequency band of interest of 1.5 to $25 \mathrm{~Hz}$. The lower frequency limit was chosen considering the lowest expected mode in $z$-axis of the structure, and the upper limit is dictated by the maximum sampling frequency of Imetrum. Further discussion on the adequacy of the chosen excitation method for extracting response characteristics is given in Section 3.2.

\subsubsection{Lux Meter}

Chauvin Arnoux CA1110 lux meter was used to record light intensity during testing. The lux meter was positioned close to the frame, as shown in Figure 1, and sampled at $1 \mathrm{~Hz}$. The expected measurement error stated by the manufacturer at the light intensity levels close to those recorded during the tests is below $1 \%$. The mean and standard deviation of light intensity recorded during the tests was $686 \mathrm{~lx}$ and $5.8 \mathrm{~lx}$, respectively. Therefore, the measurements from optics-based systems can be considered free from errors associated with the light intensity fluctuations. The recorded illuminance levels indicate the lab was well lit and represent recommended conditions for areas in which precision work is conducted [37].

\subsection{Fiducial Markers}

Different types of fiducial markers were used in conjunction with the optical instrumentation systems described in Section 2.2. In a broad sense, fiducial markers are reference objects deliberately set within the monitored system to facilitate recognition, localisation and tracking. They are often used in medicine, robotics, measurement and surveying, and X-Reality (or Cross-Reality) — a term encompassing a wide range of technology enabling realisation of virtual environments, typically in applications involving computer vision. In the context of structural vibration monitoring, light-reflective, light-emitting, and shape- 
and pattern-based fiducial markers were previously used to aid motion recognition using optical systems.

\subsubsection{Light-Reflective Markers}

Light-reflective fiducial markers comprise of a flat or spherical object covered with retroreflective paint thus bouncing the light back towards the light source, hence enhancing marker visibility. This is typically achieved with tightly-spaced 20-90 $\mu \mathrm{m}$ diameter glass spheres having rear surface covered with highly reflective coating, as seen on pictures taken with a scanning electron microscope in Figure 4. For maximum efficiency, the light source is integrated with the optical measurement system, e.g., infrared LEDs surrounding lenses of OptiTrack cameras. Light-reflective fiducial markers have been used for measurements taken in a laboratory environment [38] and in the field [39]. In this study, light-reflective fiducial markers were used to facilitate the operation of OptiTrack system described in Section 2.2.3 and enhance the performance of LDV system described in Section 2.2.4.

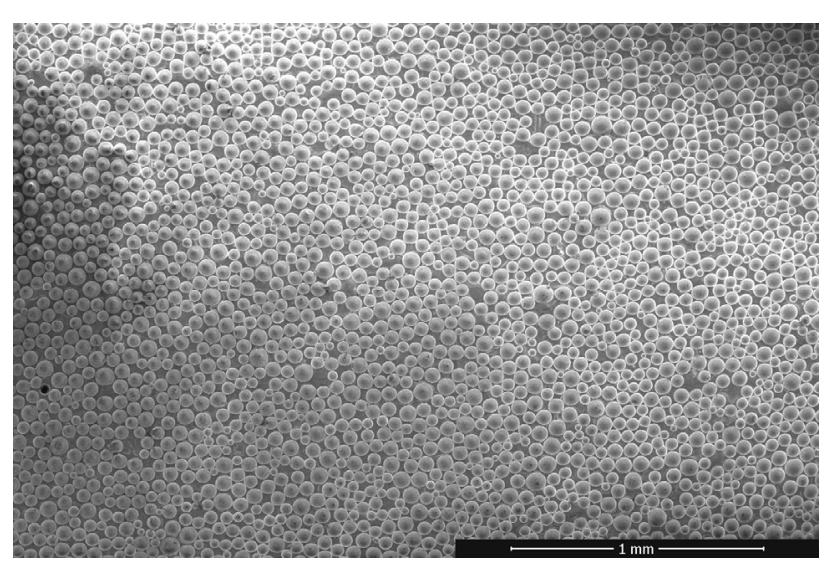

(a)

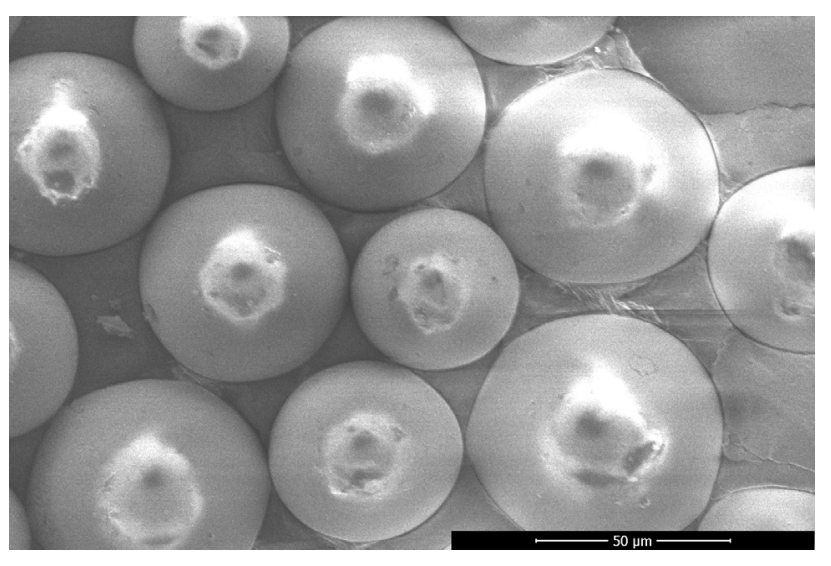

(b)

Figure 4. Light reflective marker under a scanning electron microscope with (a) 130 magnification and (b) 2000 magnification.

\subsubsection{Light-Emitting Markers}

Light-emitting fiducial markers produce light to reveal their location within captured images [40]. This approach is advocated to reduce the problem caused by insufficient and/or non-uniform structure illumination, e.g., caused by vapour interference [41]. Although markers of this type were not used in this study, they are mentioned here for completeness.

\subsubsection{Shape- and Pattern-Based Markers}

Shape and pattern-based fiducial markers are graphical objects of predefined geometry set within captured images. Shapes are 2D components of relatively simple topological structure, e.g., dots or few-sided polygons, whilst patterns are 2D assemblies of shapes. The recognition of shapes typically involves edge detection, object fitting and/or centroid computation [42]. Patterns are usually used to enable marker pose estimation, i.e., the determination of its position and orientation in 2D or 3D space [33]. Pattern-based fiducial markers were used by ArUco and template matching systems described in Section 2.2.5, and aided in area-based recognition by Imetrum system described in Section 2.2.2.

\subsection{ArUco Pose Accuracy Assessment}

Prior to the deployment of ArUco system on the structure it was necessary to establish the accuracy of the estimated pose. Furthermore, suitable marker size, camera to structure distance and angle of the camera relative to the structure (i.e., angle of incidence) had to be determined considering the camera and lens capabilities. To this end, two parallel columns of black and white ArUco square markers, varying in side size from 1 to $7 \mathrm{~cm}$ every $1 \mathrm{~cm}$, 
were printed along the two short edges of an A0 sheet attached to an inch thick fibreboard clamped against a wall, as shown in Figure 5. All markers had unique patterns and were positioned at different height such that the planar coordinates of the centres of any two markers of the same size were different but the distances between them were the same. The longest distance between a pair of markers of corresponding size measured along $x$-axis shown in Figure 5 was representative of the longest distance between ArUco markers placed on the frame during modal and initial displacement tests. The CGC was placed at various distances ( $1.5 \mathrm{~m}$ to $4 \mathrm{~m}$ every $0.5 \mathrm{~m}$ ) and facing angles ( 0 degrees, 15 degrees, and 30 degrees) to the board at the height corresponding to the middle of the sheet, represented by a yellow patch in the middle of Figure 5 . A one-minute video was recorded for each combination of the camera arrangement. The results of ArUco pose accuracy assessment are presented in Section 3.1.

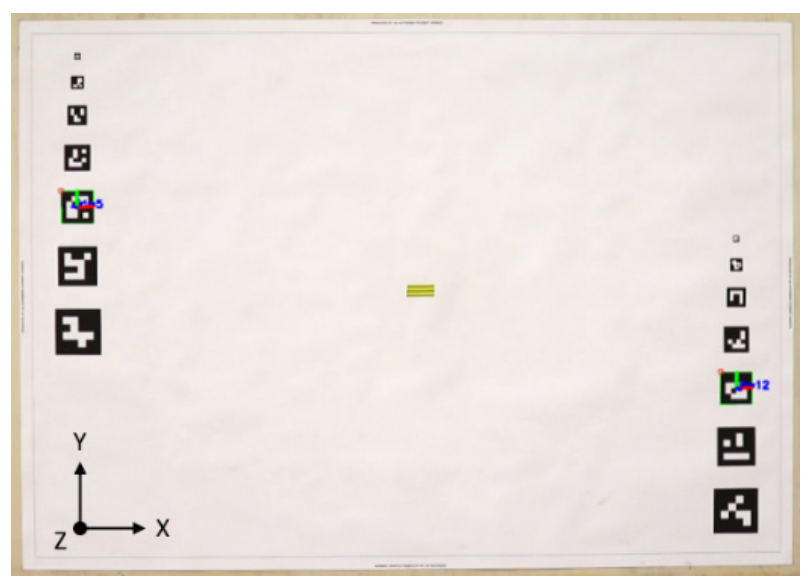

Figure 5. A screen shot of ArUco pose reconstruction accuracy test. Two markers of the same size are identified within the picture and overlaid with the local marker axes using ArUco proprietary OpenCV library.

\subsection{Modal Testing and Analysis}

The centroid of the side face of the top plate of the structure was hit 8 times with the instrumented hammer in $z$-axis to obtain correspondingly 8 windows of $64 \mathrm{~s}$ duration, containing time-synchronised structural response data from accelerometers and input force from the hammer. The length of the window was chosen such as to ensure vibration amplitudes would decay to values below observable. However, since the signals from camera-based MCS contained significant amount of noise at low vibration levels, an exponential decay window was applied to the corresponding force and response signals in each widow during modal analysis. The artificial (i.e., numerical) damping was subsequently removed to obtain unbiased modal damping estimates. The acquisition of data by all optical systems was performed in parallel, apart from LDV since it cannot be used to obtain the structural response data at more than one location at the time. Therefore, a dedicated test was run for the purpose of modal testing with LDV. For each of the 14 locations measured with LDV (2 targets per plate, for all 7 plates; see Figure 2), 3 hammer hits were applied in $z$-axis at the centroid of the side face of the top plate of the structure to obtain the corresponding average force-response spectra.

A driving point measurement, i.e., an input-output set of data obtained from spatially coincident and coaxial force and response sensors, is required in EMA to obtain mass scaled mode shapes (and determine modal mass). However, in many practical applications this is impossible to realise due to physical constraints. Hence, an alternative sensor location needs to be used. In this study, the driving point measurement relied on the response sensors located around the perimeter of the top plate, as discussed in Section 2.2 and shown in Figure 2b,c. The difference in force and response sensors' collocation is in this case acceptable since the high stiffness of the plate, together with uniform mass distribution, 
caused its lowest natural frequencies (at close to $1 \mathrm{kHz}$ as estimated from a finite element model of a plate set in SAP2000) to be much higher than those of the frame, which were of interest. The coaxial alignment of the response sensor with the force sensor, apart from the skills of the impact hammer operator, relied on either the response sensor mounting arrangement and/or the transform of coordinates. A quality check was performed to verify this procedure, by inspecting the driving point frequency response function (FRF) magnitudes in which all resonance peaks, without exceptions, should be separated by antiresonance dips (with the corresponding abrupt shifts in the FRF phase). This is opposed to the transfer point FRF, obtained from non-collocated sensors between which there is differential motion, for which antiresonances do not need to occur. This was indeed the case, for which some supporting evidence is provided in Section 3.2.

The hammer signal was recorded together with the signals from accelerometers and LDV by their respective data acquisition systems, but not with the signals from other MCS. Therefore, the following signal time-alignment procedure was applied to be able to perform EMA using image-based MCS relying on finding a match between $z$-axis accelerometer signal from the top plate and spatially correspondent signals from those MCS. The signals were first up-sampled to a common frequency of $640 \mathrm{~Hz}$. The acceleration and displacement signals were integrated and differentiated, respectively, to obtain velocity signals. The fourth-order two-way Butterworth band-pass filter with cut-off frequencies at $1 \mathrm{~Hz}$ and $20 \mathrm{~Hz}$ was applied throughout this process to minimise the errors associated with these numerical operations. The least-square error method was used to find the time lag based on the first $10 \mathrm{~s}$ of the acceleration signal measured after the impulse excitation, corresponding to the parts of response signals with the highest signal-to-noise ratio. The signals from image-based MCS were then time-aligned with the signals from accelerometers and the instrumented hammer, and truncated to match their length.

Siemens LMS SCADAS Mobile system was used for data acquisition from the accelerometers and the hammer. The hammer data were simultaneously logged by Polytec system to obtain a force signal time-synchronised with LDV signals.

The modal identification was performed using poly-reference least squares complex frequency domain algorithm [22,43-45] implemented in Siemens LMS Test.Lab ${ }^{\text {TM }} 18.2$ software under the name PolyMAX. PolyMAX has been shown to offer considerable advantages in terms of ease of use, performance speed, and reduced operator judgment dependency towards delivering high quality modal parameter estimations, even on complex data (e.g., noisy data from systems with high damping, high order, and closely spaced modes) [43]. It compares favourably with current best-of-class commercially available EMA techniques [44,46]. H1 estimator was used in the calculation of FRFs to reduce the effect of uncorrelated noise in the structural response signals. The results of modal analysis are presented in Section 3.2.

\subsection{Initial Displacement Testing and Analysis}

The initial displacement tests were conducted to assess the measurement accuracy at various motion amplitudes and the influence of the CGC angle of incidence on motion reconstruction with ArUco. The top plate was pulled approximately $5 \mathrm{~mm}$ away from its resting position in z-axis direction and then released. All optical MCS were simultaneously recording motion of the top plate of the frame for one minute at the time. The position and orientation of CGC was changed between tests, such that it was facing the side of the frame with ArUco markers at angles of 0, 15, and 30 degrees. The measurement point for LDV was chosen at the centroid of the side of the top plate facing the laser heads. The results of initial displacement tests are presented in Section 3.3.

\section{Results and Discussion}

\subsection{ArUco Pose Accuracy Results}

The error estimates are plotted in Figure 6 for all camera and markers arrangements and for all measured orthogonal directions. The error is expressed as mean magnitude, 
denoted by colour coding of the faces of the cubes, and RMS values, denoted by the length of the red lines projecting out from the cubes' faces. The information associated with each face of the cube corresponds to the measurement along the axis denoted within the parallel backdrop plane of Figure 6, as defined in Figure 5. The quality of reconstruction diminishes with increased distance of the camera from the frame, increased camera angle and decreased size of the markers. The same relationship is observed for the stability of reconstruction, expressed in terms of RMS error, which is associated with the measurement noise. No estimates were obtained for the cubes missing from the grid (see Section 2.4) for which the system arrangement was found inadequate for pose reconstruction. The least accuracy was obtained for the measurements in $z$-axis, as defined in Figure 5, which is in line with the results presented in Popescu et al. [34]. This is because ArUco markers are planar structures and the measurements along that axis, corresponding to the depth of the image, predominantly rely on the identification of their scale rather than displacement within the image plane. Any deviation of the camera angle from 0 degrees will amplify the scale difference between markers hence lead to an increased reconstruction error.
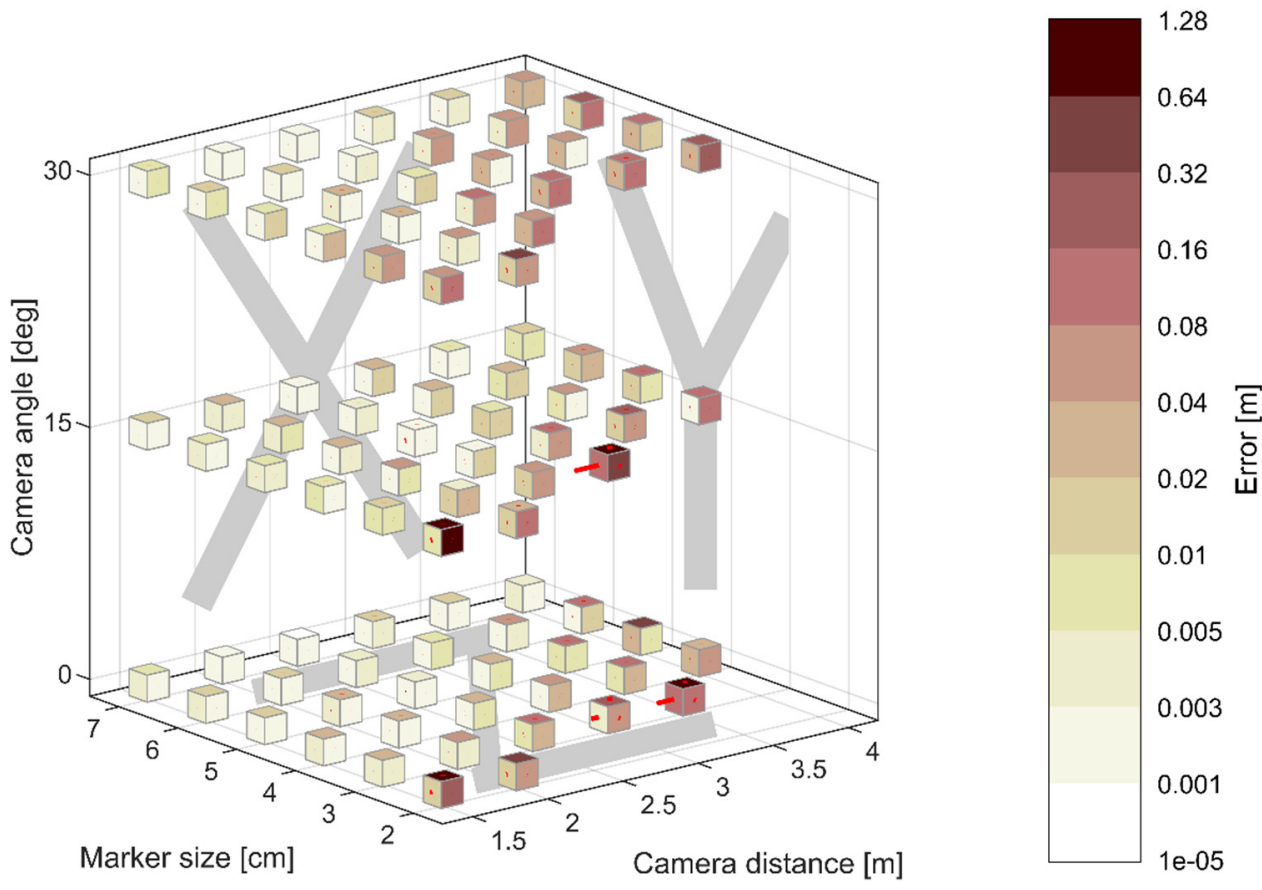

Figure 6. ArUco pose accuracy for various camera and markers arrangements in terms of mean and RMS distance error denoted by the colours of the cubes' faces and the length of lines projecting out of the cube faces, respectively. The information associated with each face of the cube corresponds to the measurement along the axis denoted within the parallel backdrop plane, as defined in Figure 5.

An appropriate camera and markers arrangement was specified considering a compromise between accuracy and applicability, in particular the availability of the area on the faces of the plates. Therefore, $5 \times 5 \mathrm{~cm}$ ArUco markers were chosen and the CGC was placed $1.5 \mathrm{~m}$ away from the structure at a zero-degree angle of incidence (i.e., facing straight on the side of the frame).

\subsection{Modal Testing Results}

The driving point FRF obtained from an accelerometer mounted at the top plate is shown in Figure 7. Six resonant peaks in Figure 7a correspond to the phase of $\pi / 2$ in Figure $7 \mathrm{~b}$, except for the last peak, close to $25 \mathrm{~Hz}$, which has relatively small amplitude. Each pair of resonant peaks is separated by an antiresonance dip, which is to be expected for driving point FRF. The correlation between the force input and the structural response 
measured with accelerometers, shown in terms of the magnitude-squared coherence in Figure $7 c$, is very good for all resonant peaks and reduces for antiresonance dips. This is to be expected since any measured response at the antiresonances, and in theory there should be none, is then dominated by uncorrelated signals, e.g., sensor noise. Taken together, the results presented in Figure 7 support the assumptions made in dispersing sensors around the plates, as discussed in Section 2.5, and show that the direct point FRF used to scale the mode shapes is reliable.

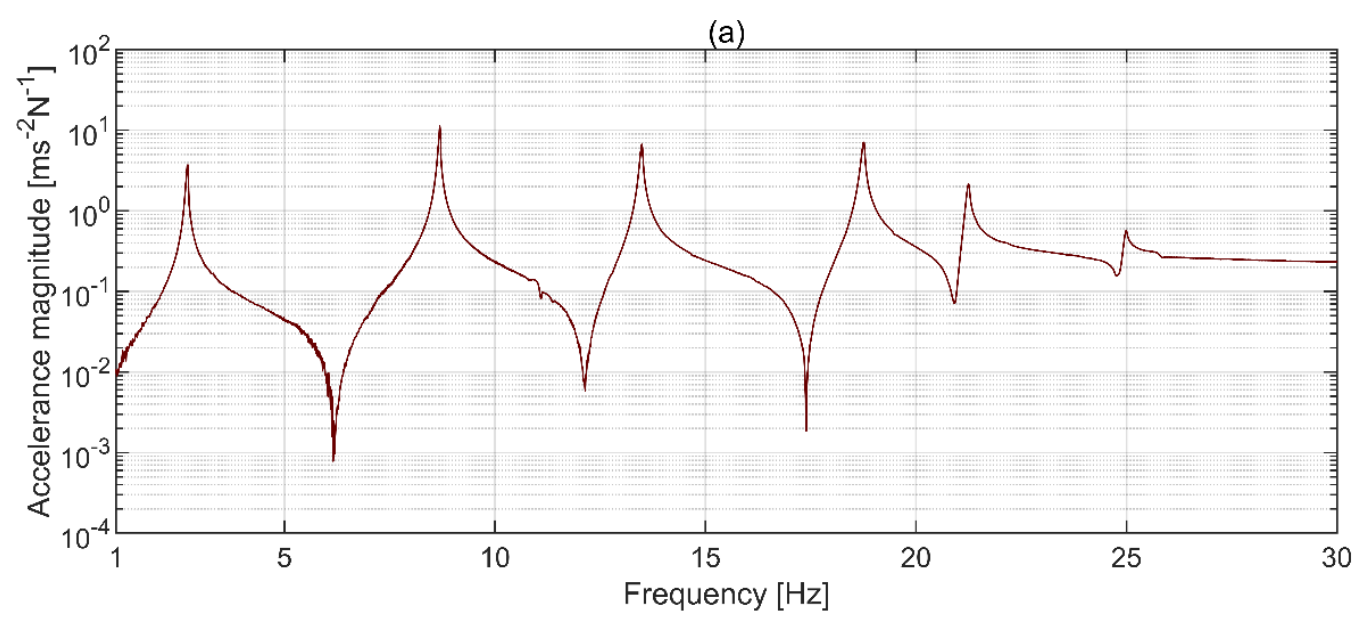

(b)

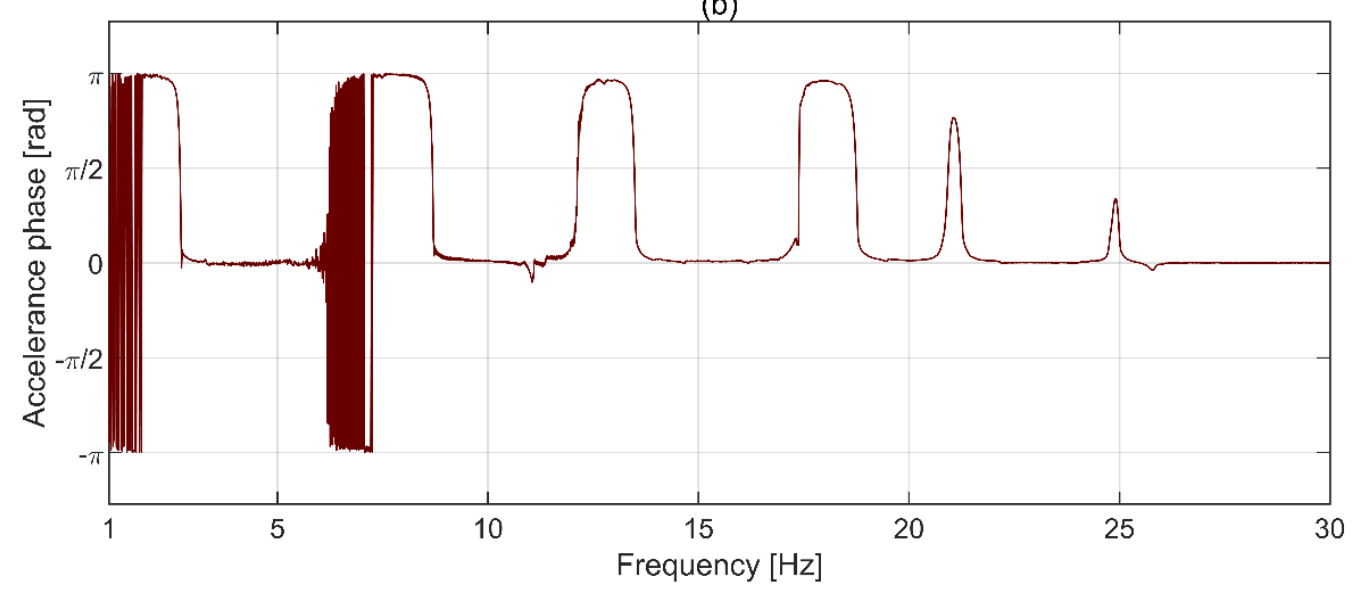

(c)

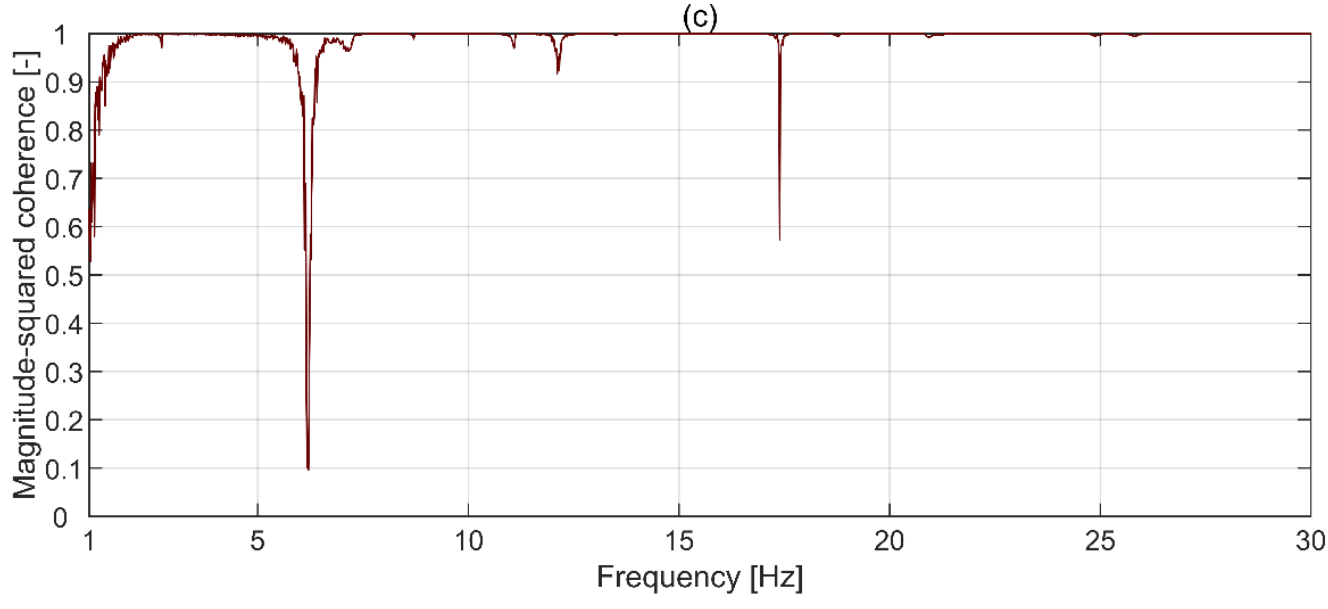

Figure 7. Driving point frequency response function (FRF) (a) magnitude, (b) phase, and (c) coherence for the accelerometer mounted at the top plate. 
The magnitudes of FRF, averaged over all measurement points and expressed in terms of mobility, are shown in Figure 8. Mobility was chosen to reconcile measurements from MCS detecting displacement (for all image-based optical systems), velocity (for LDV) and acceleration (for accelerometers). The FRF is an average over eight windows, each containing data from a single hammer impulse test, apart from LDV for which three windows were used for each measurement point (see Section 2.5). Six well-separated dominant peaks, having magnitudes above $4 \times 10^{-3} \mathrm{~ms}^{-1} \mathrm{~N}^{-1}$, are visible for accelerometry and LDV below the frequency of circa $25 \mathrm{~Hz}$, marking the Nyquist frequency for Imetrum. These peaks correspond to the lightly-damped and well-separated $z$-axis translational modes constituting the focus of this study, hereafter denoted as mode 1 to 6 . Although the behaviour of the frame above $25 \mathrm{~Hz}$ is not of interest, Figure 8 presents data up to $30 \mathrm{~Hz}$ to include the spectra roll-off for mode 6 . A less defined peak is visible at frequency of around $11 \mathrm{~Hz}$ which was identified as a torsional mode using accelerometry (i.e., by finding significant common frequency components in spectra of $\mathrm{x}$ - and $z$-axis measurements), LDV and OptiTrack, each system intrinsically capable of providing 3D motion data. Mode 6 was not recovered by both CGC-based systems, and mode 5 was not recovered by ArUco. The peak in OptiTrack data at $28.8 \mathrm{~Hz}$ was not identified as a mode.

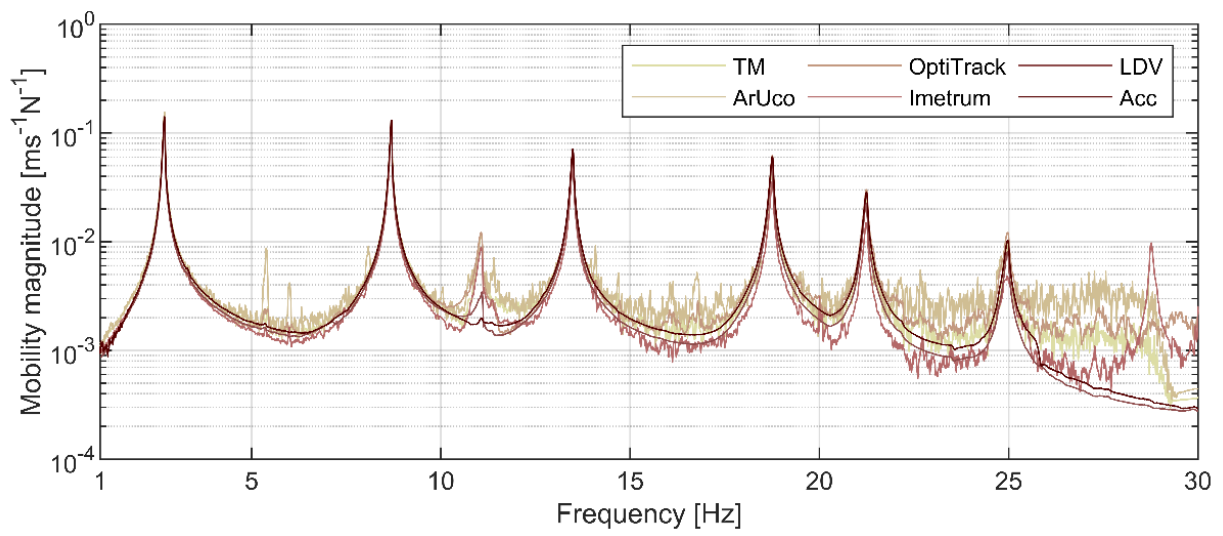

Figure 8. Averaged magnitudes of mobility FRF.

A visual inspection indicates the results from accelerometry and LDV to be seemingly compatible and relatively free from noise, which affects the performance of all other systems. The CGC-based systems are particularly prone to this problem, which is reinforced by their generally lower sensitivity.

There are slight differences in the peak amplitudes between MCS which could be attributed to systems' specification, but also the spatial origin of data. This factor is associated with the systems' operational requirements, and the requirement of having to accommodate numerous markers within each plate, as shown in Figure 2.

A complete set of numerical values for the identified modal parameters is given in Table 2 . The values in brackets represent the percentage errors relative to the measurement with accelerometers. 
Table 2. Modal parameters of the structure identified with various instrumentation systems for the first six translational modes in $z$-axis.

\begin{tabular}{|c|c|c|c|c|c|c|}
\hline & \multicolumn{6}{|c|}{ Modal Frequency [Hz] } \\
\hline & Mode 1 & Mode 2 & Mode 3 & Mode 4 & Mode 5 & Mode 6 \\
\hline Accelerometry & $2.681(\mathrm{~N} / \mathrm{A})$ & $8.673(\mathrm{~N} / \mathrm{A})$ & $13.471(\mathrm{~N} / \mathrm{A})$ & $18.730(\mathrm{~N} / \mathrm{A})$ & $21.223(\mathrm{~N} / \mathrm{A})$ & $24.943(\mathrm{~N} / \mathrm{A})$ \\
\hline LDV & $2.676(-0.20 \%)$ & $8.671(-0.02 \%)$ & $13.462(-0.07 \%)$ & $18.723(-0.04 \%)$ & $21.214(-0.04 \%)$ & $24.938(-0.02)$ \\
\hline OptiTrack & $2.680(-0.04 \%)$ & $8.675(0.02 \%)$ & $13.471(0.00 \%)$ & $18.737(0.03 \%)$ & $21.227(0.02 \%)$ & $24.949(0.03 \%)$ \\
\hline Imetrum & $2.680(-0.06 \%)$ & $8.676(0.03 \%)$ & $13.473(0.01 \%)$ & $18.737(0.04 \%)$ & $21.221(-0.01 \%)$ & $24.985(0.17 \%)$ \\
\hline ArUco & $2.681(-0.01 \%)$ & $8.675(0.02 \%)$ & $13.473(0.01 \%)$ & $18.741(0.06 \%)$ & $\mathrm{N} / \mathrm{A}$ & N/A \\
\hline \multirow[t]{3}{*}{ Template matching } & $2.680(-0.05 \%)$ & $8.675(0.02 \%)$ & $13.472(0.01 \%)$ & $18.738(0.04 \%)$ & $21.218(-0.02 \%)$ & $\mathrm{N} / \mathrm{A}$ \\
\hline & \multicolumn{6}{|c|}{ Modal damping ratio [\%] } \\
\hline & Mode 1 & Mode 2 & Mode 3 & Mode 4 & Mode 5 & Mode 6 \\
\hline Accelerometry & $0.706(\mathrm{~N} / \mathrm{A})$ & $0.224(\mathrm{~N} / \mathrm{A})$ & $0.251(\mathrm{~N} / \mathrm{A})$ & $0.227(\mathrm{~N} / \mathrm{A})$ & $0.238(\mathrm{~N} / \mathrm{A})$ & $0.233(\mathrm{~N} / \mathrm{A})$ \\
\hline LDV & $0.710(0.54 \%)$ & $0.269(20.14 \%)$ & $0.243(-3.24 \%)$ & $0.234(3.05 \%)$ & $0.241(1.46 \%)$ & $0.235(0.70 \%)$ \\
\hline OptiTrack & $0.754(6.79 \%)$ & $0.250(11.77 \%)$ & $0.246(-2.03 \%)$ & $0.206(-9.25 \%)$ & $0.184(-22.77 \%)$ & $0.110(-52.73 \%)$ \\
\hline Imetrum & $0.704(-0.31 \%)$ & $0.245(9.29 \%)$ & $0.227(-9.59 \%)$ & $0.210(-7.52 \%)$ & $0.203(-14.76 \%)$ & $0.083(-64.23 \%)$ \\
\hline ArUco & $0.707(0.21 \%)$ & $0.226(0.93 \%)$ & $0.176(-29.95 \%)$ & $0.130(-42.47 \%)$ & $\mathrm{N} / \mathrm{A}$ & $\mathrm{N} / \mathrm{A}$ \\
\hline \multirow[t]{3}{*}{ Template matching } & $0.735(4.13 \%)$ & $0.249(11.51 \%)$ & $0.237(-5.47 \%)$ & $0.200(-11.68 \%)$ & $0.158(-33.54 \%)$ & $\mathrm{N} / \mathrm{A}$ \\
\hline & \multicolumn{6}{|c|}{ Generalised mass [kg] } \\
\hline & Mode 1 & Mode 2 & Mode 3 & Mode 4 & Mode 5 & Mode 6 \\
\hline Accelerometry & $19.55(\mathrm{~N} / \mathrm{A})$ & $20.74(\mathrm{~N} / \mathrm{A})$ & $17.71(\mathrm{~N} / \mathrm{A})$ & $15.55(\mathrm{~N} / \mathrm{A})$ & $13.80(\mathrm{~N} / \mathrm{A})$ & $10.78(\mathrm{~N} / \mathrm{A})$ \\
\hline LDV & $18.05(-7.66 \%)$ & $17.47(-15.78 \%)$ & $18.11(2.27 \%)$ & $15.44(-0.72 \%)$ & $15.26(10.59 \%)$ & $10.31(-4.38 \%)$ \\
\hline OptiTrack & $17.57(-10.11 \%)$ & $17.83(-14.04 \%)$ & $14.27(-19.41 \%)$ & $13.95(-10.26 \%)$ & $14.45(4.71 \%)$ & $17.13(58.92 \%)$ \\
\hline Imetrum & $18.58(-4.93 \%)$ & $20.18(-2.72 \%)$ & $20.72(16.98 \%)$ & $23.58(51.65 \%)$ & $24.25(75.76 \%)$ & $26.95(149.99 \%)$ \\
\hline ArUco & $17.85(-8.65 \%)$ & $20.13(-2.92 \%)$ & $28.42(60.50 \%)$ & $32.12(106.6 \%)$ & N/A & N/A \\
\hline Template matching & $18.02(-7.83 \%)$ & $19.06(-8.07 \%)$ & $19.11(7.93 \%)$ & $18.28(17.57 \%)$ & $21.43(55.34 \%)$ & $\mathrm{N} / \mathrm{A}$ \\
\hline
\end{tabular}

For better readability, the errors in modal parameter estimation are presented in a graphical way in Figure 9. The data not recovered by ArUco and template matching are indicated with crosses. As could be expected, the accuracy of frequency reconstruction presented in Figure $9 \mathrm{a}$ is overall high with the error magnitude below $0.2 \%$. The discrepancy between results can be mainly attributed to the various sampling rates used by MCS, as stated in Table 1, hence various resolution of data in the frequency domain. The higher frequency resolution for LDV relative to accelerometry, imposed by the system requirements (i.e., the lowest sampling rate available in the LDV proprietary software), is the most likely reason for LDV underestimating modal frequencies across the whole range.

The identified damping levels are representative of those measured on bridges, chimneys, and steel masts and towers [24,47-51].

The accuracy of damping and generalised mass reconstruction, presented in Figure 9b,c, respectively, shows high variability between modes and within each mode above the 2nd mode. As could be expected from the system specifications, the highest overall accuracy relative to accelerometry is recorded for LDV. The relatively high difference in damping estimates for mode 2 is probably also related to the different ability of the instrumentation systems to capture the neighbouring torsional mode at approximately $11 \mathrm{~Hz}$, as seen in Figure 8. In this particular case, the results obtained for LDV are more reliable. For all MCS based on image processing, the accuracy diminishes with the mode rank. The bias error was minimised by choosing the measurement duration allowing signals to decay to values below observable, considering the sensitivity of MCS, and using an exponential weighting window on both force and response measurements, hence minimising leakage. The random error in FRF obtained with H1 estimator, assuming no extraneous noise on the hammer force input (see Figure 3), provided by the formula given in Bendat and Piersol [52] is negligibly small for the recovered modes. Therefore, this systematic behaviour should be attributed to insufficient MCS sensitivity and relatively high noise levels obtained by taking the average from only eight windows. This can be understood by closer inspection of the magnitude of compliance FRF for ArUco system and the corresponding magnitudesquared coherence shown in Figure 10. The data were obtained by taking an average over eight windows, each containing data from a single hammer impulse test, and by applying 
an exponential window on both signals with the decay constant of -4 , thus ensuring the window reduces to below $2 \%$ of its initial value of unity at the end of the record. The compliance FRF was chosen in this case as it relies on the displacement signal, which is native to ArUco. Four distinctive peaks are visible in the spectrum of compliance FRF magnitude above $4 \times 10^{-4} \mathrm{mN}^{-1}$ in Figure 10a, which correspond to the four lowest translational modes in $z$-axis of the frame. The peaks for mode 5 and 6 are hardly visible in the spectrum and mostly buried in the uncorrelated noise having magnitude reaching up to approximately $7 \times 10^{-5} \mathrm{mN}^{-1}$ within the corresponding frequency band. As shown in Figure 10b, the coherence is acceptable for the first four modes, but drops to unacceptable levels for frequencies associated with mode 5 and 6, for which the magnitude FRF in Figure $10 \mathrm{a}$ is heavily distorted between resonant peaks. In comparison, the mobility FRF magnitude for accelerometers in Figure 8, from data sampled at a similar rate to CGC systems (see Table 1), is very clear and there is no distortion throughout the whole frequency bandwidth.

Interestingly, the performance of Imetrum and template matching in terms of modal frequency, damping and generalised mass is comparable up to the 4th mode at circa 18.73 $\mathrm{Hz}$. The two MCS relied on different fiducial markers and Imetrum used two cameras whilst template matching used only one camera. Furthermore, the results from Imetrum were averaged over two markers tracked within the top plate, whilst a single marker was tracked by template matching. Beyond the 4th mode, all image-based MCS yield little accuracy in terms of damping and generalised mass reconstruction.

(a)

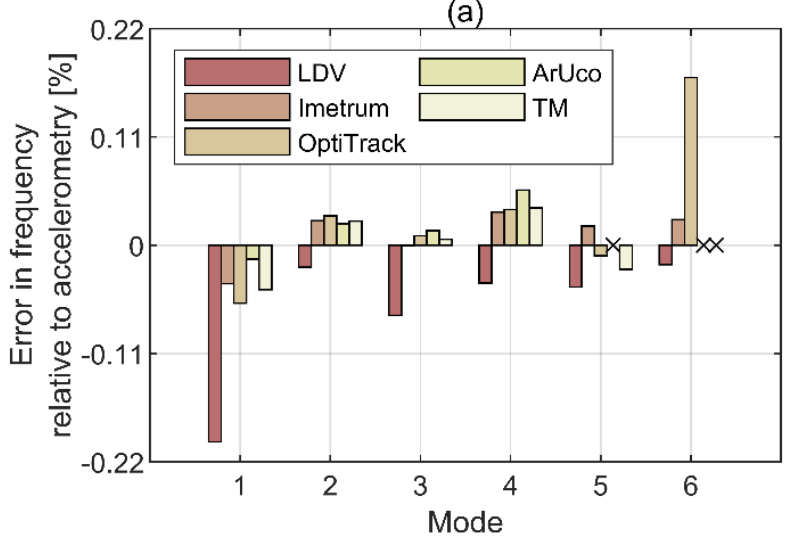

(b)

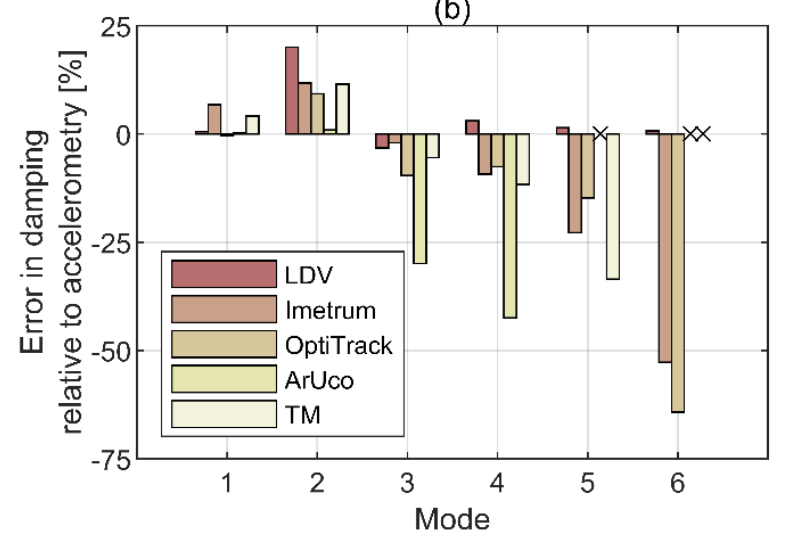

(c)

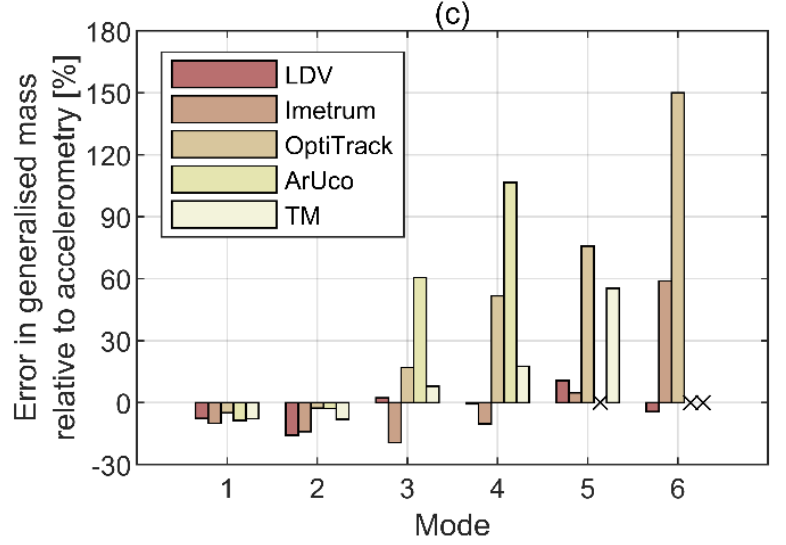

Figure 9. Errors in modal parameters relative to accelerometry in terms of (a) modal frequency, (b) damping, and (c) generalised mass. The data not recovered by ArUco and template matching systems are represented by crosses. 
(a)

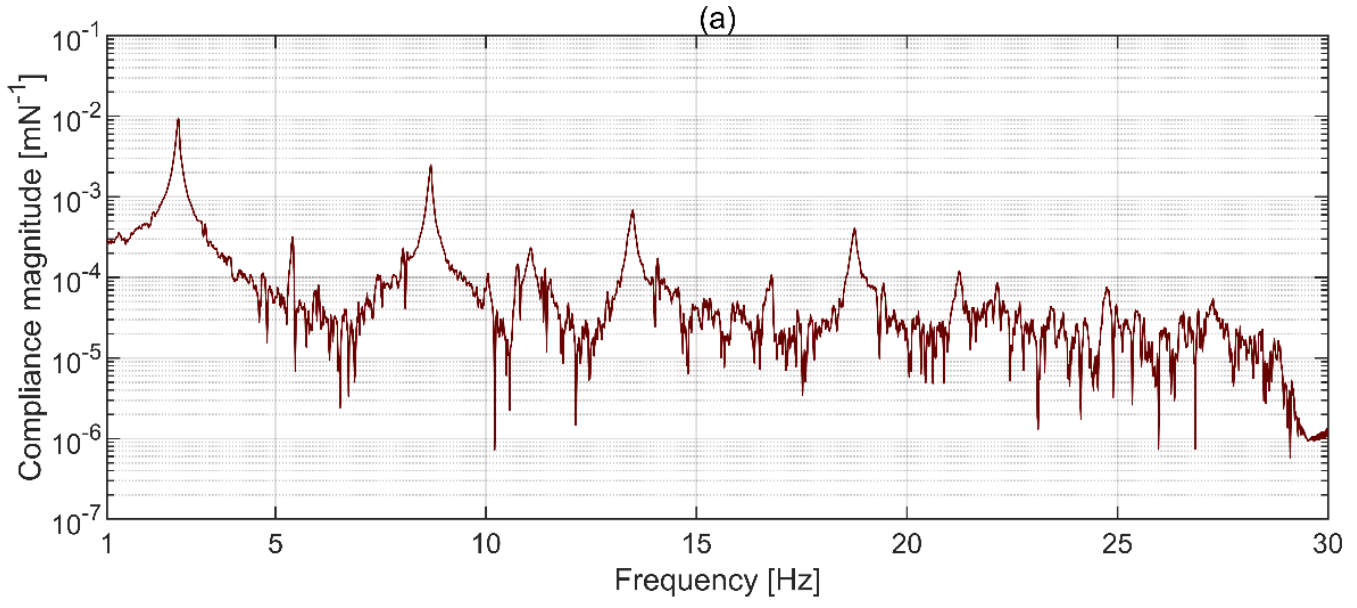

(b)

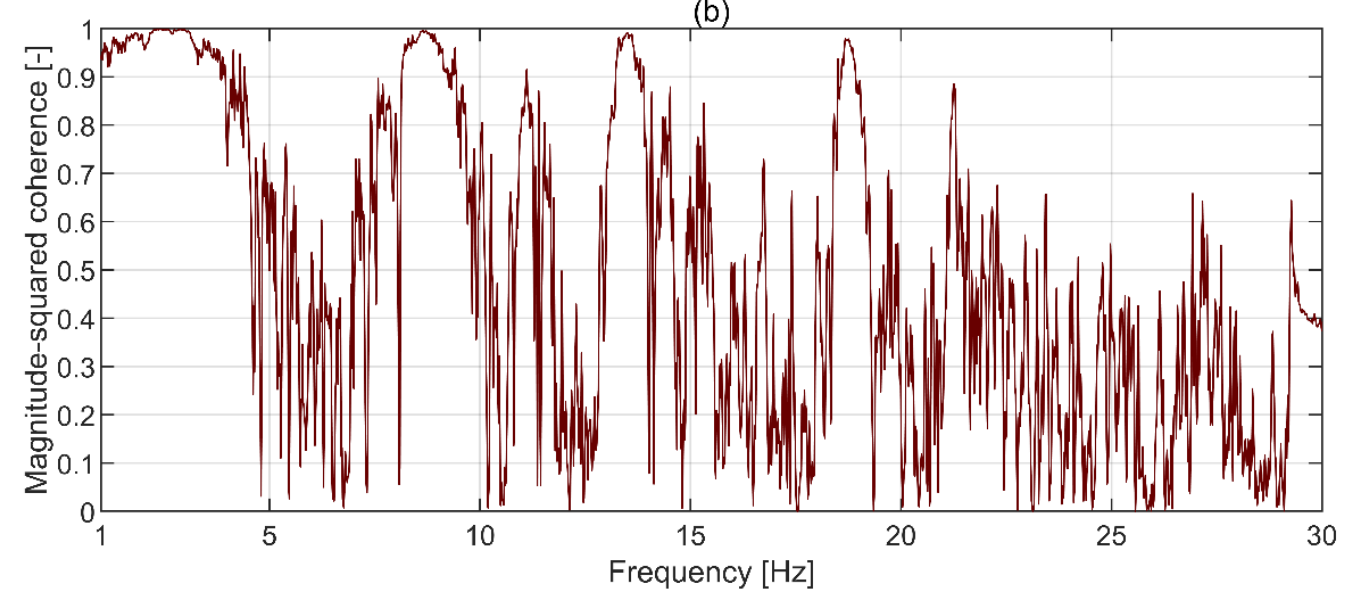

Figure 10. (a) Magnitude of compliance FRF and (b) corresponding magnitude-squared coherence for measurements with ArUco.

Another reason for the deficiency in reconstruction of mode 5 and 6 by CGC-based MCS is revealed when considering relatively low response levels for these modes at the top plate, as shown in Figure 8, together with the mode shapes, shown in Figure 11. For clarity of presentation, the mode shapes in Figure 11 are normalised to the maximum magnitude of unity. Although the relative resolution of mode shapes coarsens with the increasing mode shape rank, the relative movement at the measurement points is what could be expected from the type of tested structure when idealised as a lumped-mass model. The match between mode shapes recovered with different MCS seems generally good. However, the modal displacement at the top plate, coinciding with the point of application of the hammer force, is relatively small for mode 5 and 6 . This means that the energy transmitted to these modes, bearing in mind the force level was similar across the whole frequency range of interest, could have been insufficient to excite them beyond the noise floor of image-based MCS. This also explains low coherence for mode 5 and 6 in Figure 10b.

Figure 12 presents modal assurance criterion (MAC) [53,54] calculated to quantify the correlation between mode shapes for the first six translational modes. Overall, MAC obtained against accelerometry for all MCS is above 0.987 which, for all practical purposes, can be considered satisfactory. The most consistent agreement with accelerometry for mode 6 is found for LDV. The MAC for mode 6 for the other two systems which recovered this mode shape, i.e., Imetrum and OptiTrack, deteriorates significantly relative to the MAC for lower rank modes. 


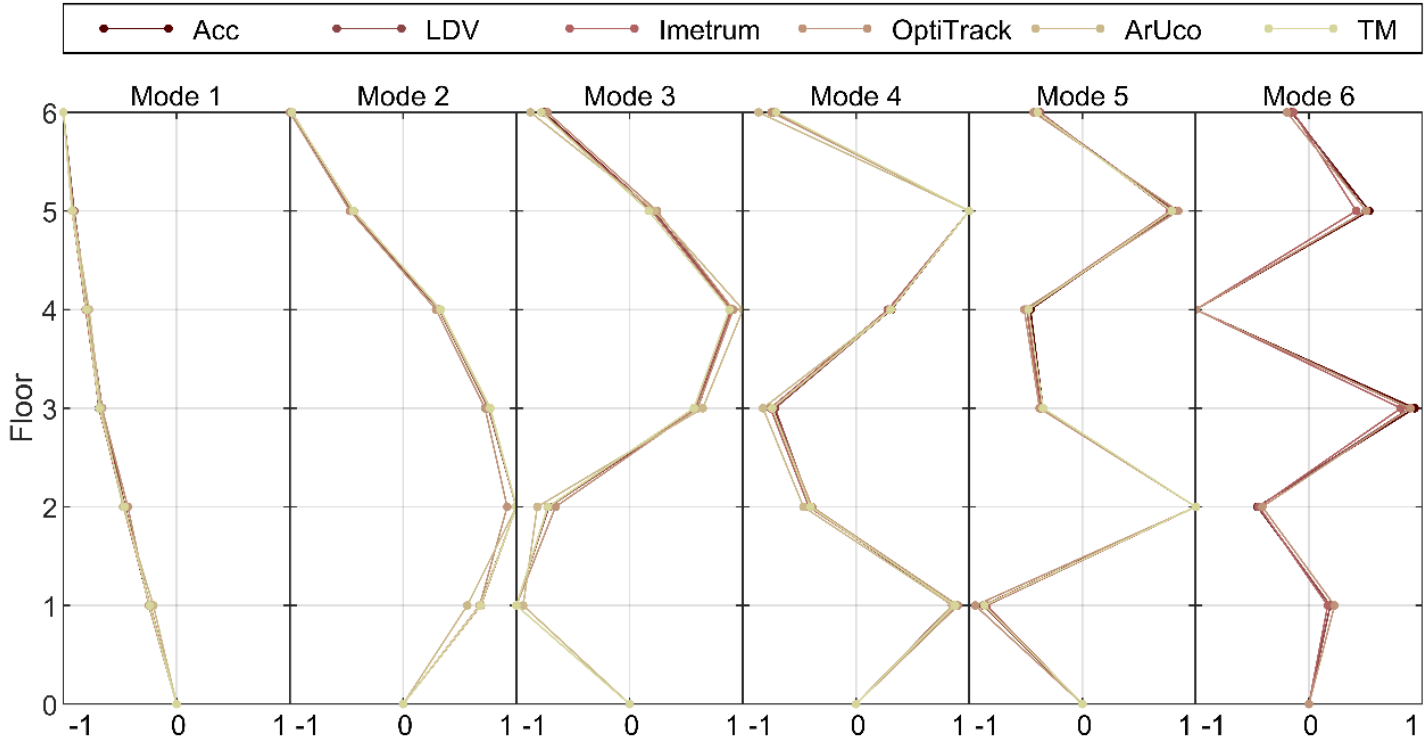

Figure 11. The first six translational mode shapes of the frame in $z$-axis.
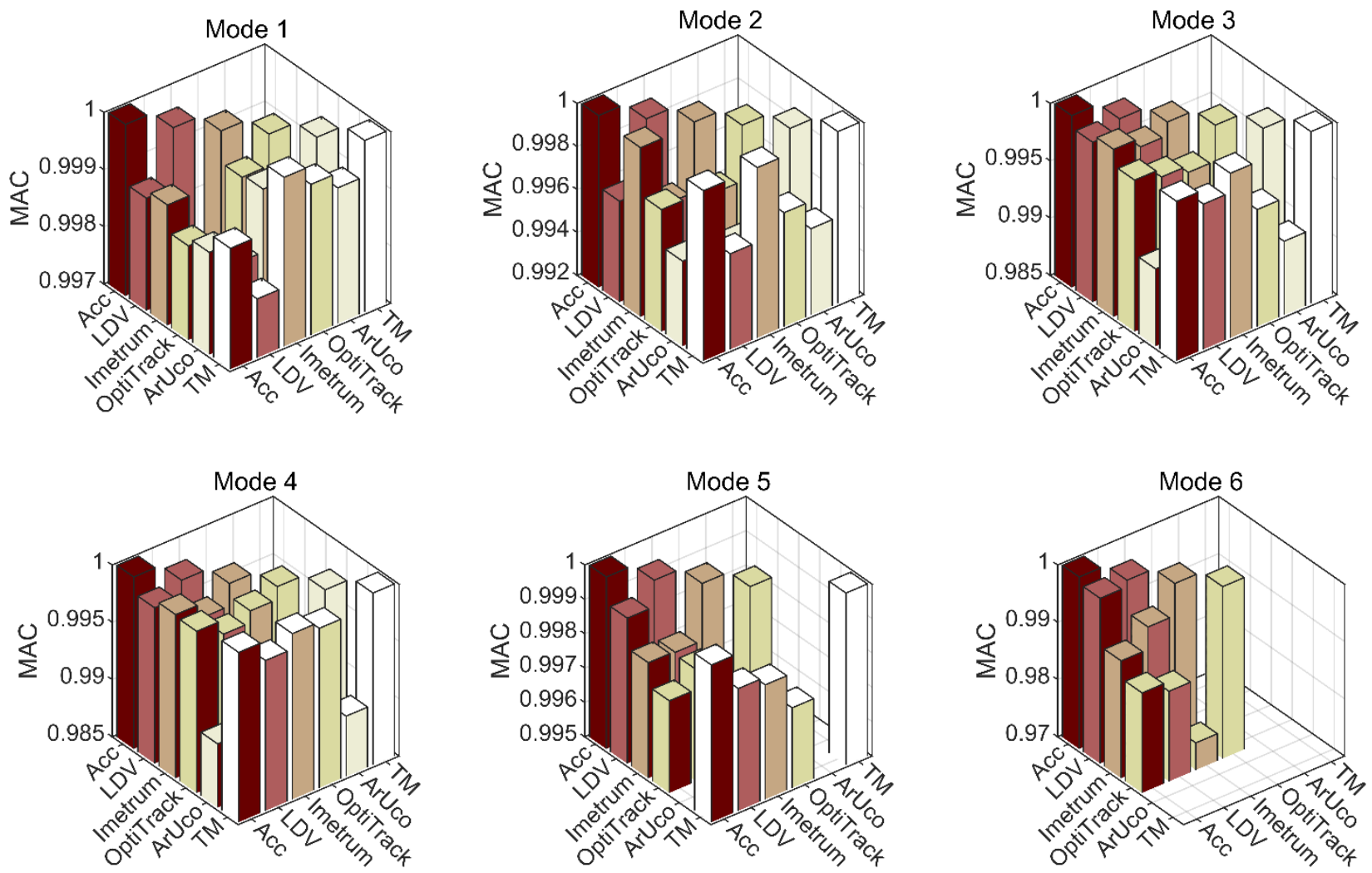

Figure 12. Modal assurance criterion (MAC) based on eigenvectors obtained from data recorded with all motion capture systems (MCS) for the first six translational mode shapes. The scale of the vertical axis is adjusted such as to contain all available results.

The correlation of mode shapes obtained with ArUco with those obtained with accelerometry deteriorates consistently with the mode shape rank. This is clearly visible in Figure 13, which is a snapshot of the results presented in Figure 12. 


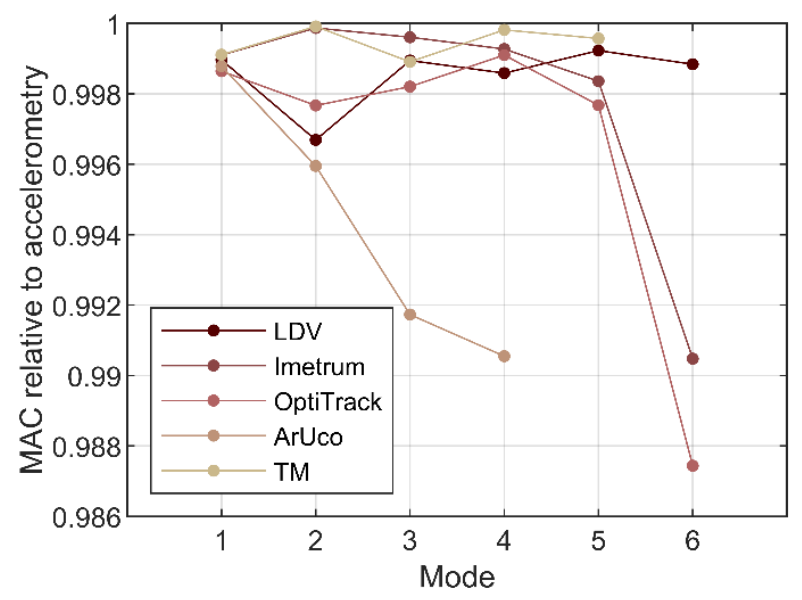

Figure 13. Modal assurance criterion (MAC) for all optical MCS relative to accelerometry.

In conclusion to this section, the benchmark estimates of modal parameters are assumed here to come from accelerometry and LDV, since they give corresponding results in terms of the modal frequency, damping and generalised mass, as seen in Table 2 and Figure 9, and the mode shape, as seen Figures 11 and 12. This is the case for all translational modes considered in this study, with the exception of mode 2 of which identification is affected by the proximity of a torsional mode. All image-based MCS give corresponding results for the lowest modes, but the error between estimates of modal parameters increases with the mode rank.

The errors in modal parameters obtained with image-based MCS are not out of range with results reported from an exercise aiming to assess the consistency in modal identification of a structure moved between twelve European laboratories [55]. The variability in the estimated modal frequency, damping and generalised mass, obtained from accelerometry, was within $4 \%, 30 \%$, and $10 \%$, respectively.

The performance of image-based commercial MCS—Vicon (similar to OptiTrack used herein), based on three cameras and light-reflective markers, was compared to laser displacement sensors (LDS) in Park et al. [56]. The measurements were taken from a scaled model of a pylon subjected to uniform wind flow within a wind tunnel. The frequency and damping estimates obtained from LDS for the first translational mode were $6.93 \mathrm{~Hz}$ and $0.359 \%$, respectively. The corresponding estimates from Vicon were within $0.3 \%$ and $177 \%$, respectively. In a subsequent study [24], the performance of Vicon, consisting of four cameras, was compared to accelerometry and LDS. The behaviour of a scaled model of a 3D building frame having three storeys (rather than six storeys as in the model used herein) and stiffened with bracing in one lateral direction to enforce dominant vibrations in the other lateral direction, in response to an initial displacement, was of interest. There were no errors, up to two decimal places, in the frequencies of three translational modes between $1.07 \mathrm{~Hz}$ and $5.15 \mathrm{~Hz}$ and the error in modal damping, expected to fall within the range of $0.2 \%$ to $0.84 \%$, was within approximately $2 \%$. However, none of these studies reported modal mass estimates nor conducted EMA.

An alternative way to present the results obtained herein would be to normalise mode shapes by the modal mass rather than the maximum amplitude at the measurement points. The MAC obtained from such normalised mode shapes would remain the same yet the modes themselves would carry the same error with the reported modal mass. However, this would mask the fact that the relative movement of measurement points on the tested structure is consistent in scale between all deployed instrumentation systems.

\subsection{Initial Displacement Testing Results}

Having established that image-based MCS provide modal parameters compatible with benchmark estimates from accelerometry and LDV for mode 1 and 2, the focus in this 
section is on these two modes only. The LDV data were used herein as a benchmark to avoid numerical errors associated with double-integration of the acceleration data and to account for better performance of this system in capturing characteristics of mode 2 . All MCS data were up-sampled to a common frequency of $3 \mathrm{kHz}$ for precise time alignment.

\subsubsection{Vibration Amplitude}

The envelopes of the amplitude of peak displacement identified within each vibration cycle for the first two modes are shown in Figure 14. The data were obtained by applying a two-way fourth-order Butterworth band-pass filter with cut off frequencies $\pm 0.15 \mathrm{~Hz}$ from the identified modal frequencies. The ArUco signal used in the comparison was recorded at 0 degrees CGC incidence angle relative to the frame (see Figure 2). It can be seen that all four image-based MCS are capable of measuring dynamic displacements with sub-millimetre (and sub-pixel) accuracy. Visual inspection indicates that the motion of the top plate in mode 1, shown in Figure 14a, is recovered with good accuracy for all image-based MCS relative to the benchmark LDV data. For mode 2, the signals from Imetrum and ArUco, shown in Figure 14b, diverge from the benchmark LDV data for amplitudes below $0.05 \mathrm{~mm}$. However, it needs to be noted that the motion amplitudes recorded for mode 1 and 2 differ by more than an order of magnitude. The RMS errors in peak displacement amplitudes relative to LDV are given in Table 3.

(a)

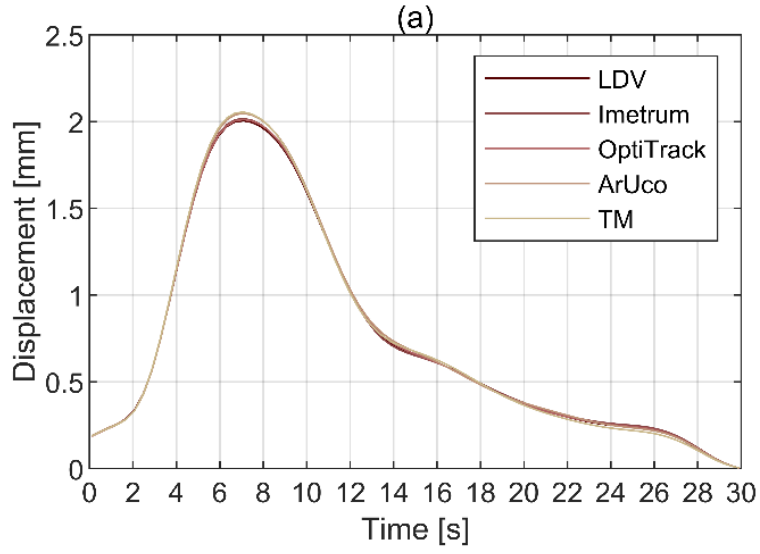

(b)

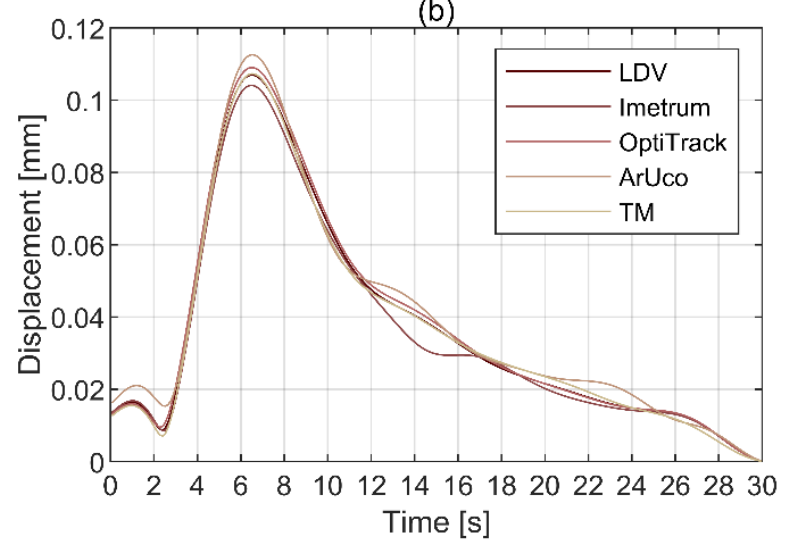

Figure 14. Envelopes of the amplitudes of peak displacement for (a) mode 1 and (b) mode 2.

Table 3. RMS errors in the amplitudes of peak displacement for mode 1 and mode 2 relative to LDV.

\begin{tabular}{cccc}
\hline \multirow{2}{*}{ MCS } & Number of Cameras & Mode 1 & Mode 2 \\
\cline { 3 - 4 } & & RMS Error [mm] & RMS Error [mm] \\
\hline OptiTrack & 8 & $6.74 \times 10^{-3}$ & $1.23 \times 10^{-3}$ \\
Imetrum & 2 & $7.15 \times 10^{-3}$ & $2.61 \times 10^{-3}$ \\
ArUco & 1 & $1.74 \times 10^{-2}$ & $3.12 \times 10^{-3}$ \\
Template matching & 1 & $1.98 \times 10^{-2}$ & $1.34 \times 10^{-3}$ \\
\hline
\end{tabular}

The RMS errors are generally small for all MCS, falling below $0.02 \mathrm{~mm}$. The best match with LDV is found for OptiTrack. Although the accuracy generally improves with the number of cameras in MCS (see Table 1), overall, the performance of MCS relying on a single CGC either matches that of multi-camera systems or is not out of range by much.

\subsubsection{Influence of the Angle of Incidence of CGC}

A dedicated set of tests was conducted to establish the influence of the CGC angle of incidence on the accuracy of vibration measurement with ArUco. The CGC was set at three angles of incidence: 0,15 , and 30 degrees. The results of these tests, together with the benchmark data from LDV, are presented in Figure $15 \mathrm{a}-\mathrm{c}$, respectively. It can be seen that 
all angles other than 0 degrees generate significant errors. This is predominantly caused by the motion component out of plane relative to the camera and is consistent with the results of static pose reconstruction discussed in Section 3.1.

(a)

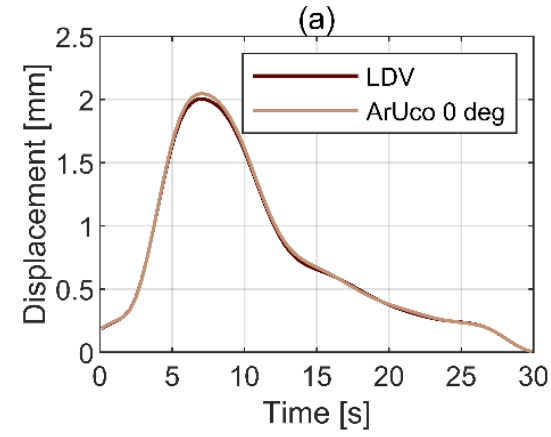

(b)

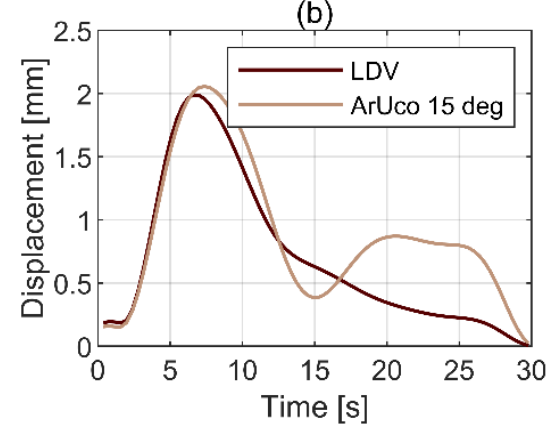

(c)

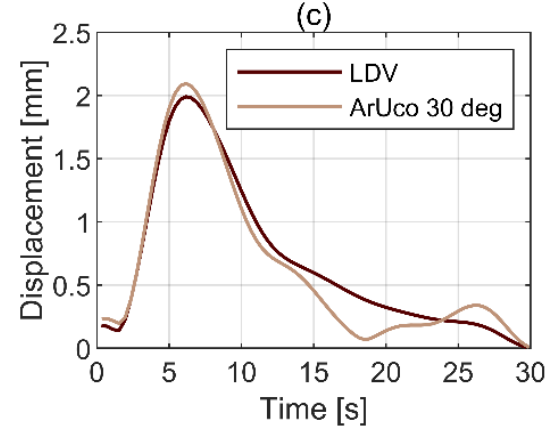

Figure 15. ArUco reconstruction of motion amplitude for mode 1 depending on the CGC angle of incidence: (a) 0 degrees, (b) 15 degrees, (c) 30 degrees.

\subsection{Limitations of This Study and Deployment Considerations on In Situ Structures}

The way various systems were set up is of course specific to the conducted tests. Although every effort was made to make informed decisions and apply best judgement, it is possible that the systems were not operated at their optima. For example, the choice of fiducial markers and, to some extent, their location on the structure, as explicitly studied elsewhere [7], was often arbitrary, dictated by suggestions reported in the literature and authors' own experience.

There are certain limitations and difficulties in using optical MCS beyond those related to their intrinsic design, which, ultimately, can lead to the reduced quality measurements in structural vibration monitoring. These factors include uncertainty in system calibration, environmental effects, and deployment difficulties [1]. For example, light intensity fluctuations during monitoring, e.g., those associated with alternating cloud coverage, and light refraction can lead to drift and sudden discontinuities in the measured signals. These problems can be minimised in post processing, e.g., with zero-normalised versions of certain correlation criteria for template matching method [57]. Changes in atmospheric pressure and wind speed can be prevalent [5]. Camera instability or drift associated with settlement can be reduced by ensuring rigid camera mounting [58] or using an auxiliary reference point within the captured image, stationary in the absolute reference frame, and subtracting its motion from the measured structural displacement [6]. Camera vibration can also be compensated for, to some extent, by incorporating an accelerometer recording camera motion for use in post processing [59]. However, most of these challenges, which are often coincident, cannot be completely eliminated during in situ investigations on outdoor structures. Therefore, to obtain a benchmark performance of optical structural vibration monitoring systems, a controlled environment is required, which shelters them from the elements. This was achieved in the current study.

\section{Conclusions}

The performance of five optical motion capture systems (MCS) in experimental modal analysis (EMA) is investigated in this study, based on the data obtained from a scaled model of a frame of a 3D building. This includes Polytec laser Doppler vibrometer-LDV, two commercial multi-camera systems-OptiTrack and Imetrum, and two open-source systems utilising a single consumer-grade camera (CGC) based on feature- and area-based template matching-ArUco and TM, respectively. The results obtained with accelerometry are used as a validating benchmark. A complete set of modal parameters is considered, including modal frequency, mode shape, modal damping and modal (generalised) mass. 
To the best of authors' knowledge, this experimental study constitutes the broadest to date comparative effort towards understanding the performance of optical MCS in EMA.

Dictated by the specification of some of the deployed MCS, the translational response of the frame associated with the weak axis of the columns is of interest, up to the frequency of $25 \mathrm{~Hz}$. Six translational modes are identified by all systems, apart from ArUco which could not recover mode 5 at $21.2 \mathrm{~Hz}$ and mode 6 at $24.9 \mathrm{~Hz}$, and TM which could not recover mode 6 . The maximum discrepancy in modal frequency is below $0.2 \%$ and can be explained by different sampling rates inherent to the deployed MCS. The modal assurance criterion calculated for the empirical mode shapes does not fall below 0.987 .

The modal parameters derived from LDV are in excellent agreement with accelerometry for all considered modes. This is apart from the damping for mode 2, of which reconstruction is affected by the neighbouring torsional mode, which is identified with greater accuracy by LDV rather than accelerometry. All image-based MCS, i.e., OptiTrack, Imetrum, ArUco and TM, can measure the displacement with sub-millimetre and sub-pixel accuracy. A good match with a benchmark measurement from LDV is found for amplitudes down to $0.05 \mathrm{~mm}$. Considering all image-based MCS, the approximate maximum error magnitude in modal damping and mass is, respectively, $7 \%$ and $10 \%$ for mode 1 at $2.7 \mathrm{~Hz}$, and $12 \%$ and $14 \%$ for mode 2 at $8.7 \mathrm{~Hz}$. All modal parameters for mode 3 at $13.5 \mathrm{~Hz}$ and mode 4 at $18.7 \mathrm{~Hz}$ are recovered reasonably well by all systems apart from ArUco, with the maximum error in modal damping and mass below $12 \%$ and $20 \%$, respectively. Another exception is Imetrum for which the error in modal mass reconstruction for mode 4 is above $50 \%$. The performance of all image-based MCS in the reconstruction of modal damping and mass for mode 5 and 6 is unsatisfactory. This is caused by a combination of factors, most notably low response levels at these modes, relatively low sensitivity and high noise floor of these MCS, and mode 6 being close to the Nyquist frequency.

ArUco system, relying on feature-based template matching, performs best with the CGC positioned at 0 degrees angle of incidence. This is found in static pose reconstruction tests and in vibration tests. The more the angle of incidence deviates from 0 degrees, the higher measurement error. When implemented with CGC at its preferable orientation, an area-based template matching algorithm can match the performance of Imetrum, relying on two cameras, in the lowest four modes. The error magnitude in modal damping and mass is then below $20 \%$.

In general, image-based MCS do not match the performance of conventional accelerometers nor LDV in terms of sensitivity and noise levels. Therefore, for maximum efficiency, the organisation of instrumentation systems during the tests and the testing protocols needs to be carefully considered to ensure all modes of interest are sufficiently excited by the force source and can be recovered in modal analysis. Since it is always a good practice to conduct a survey to identify a suitable arrangement of instrumentation prior to any modal test, the penalty associated with this task may not be significant.

Overall, the presented results suggest that MCS based on a single consumer-grade camera and open-source tracking algorithm is capable of providing data enabling EMA to be conducted when frequencies of interest fall below approximately $20 \mathrm{~Hz}$, which is the case for many civil engineering structures. The main advantage of a system of this type is in the ease of deployment and economy - the cost, which can be limited to hardware only, is a small fraction of that associated with commercial systems. Therefore, the outcomes of this study encourage further efforts in exploring the performance and optimisation, e.g., through hybrid solutions, of image-based optical motion capture systems in the context of fully detailed EMA, rather than the identification of modal frequencies and mode shapes only.

Author Contributions: Conceptualization, M.B.; methodology, M.K. and M.B.; software, M.K.; formal analysis, M.K. and M.B., resources, M.B. and N.N.; writing-original draft preparation, M.K., M.B. and N.N.; writing-review and editing, M.B. and N.N.; supervision, M.B. and N.N.; funding acquisition, M.K., M.B. and N.N. All authors have read and agreed to the published version of the manuscript. 
Funding: M.K. was supported by the Bolashak International Scholarship of the President of the Republic of Kazakhstan for doctoral studies at the University of Leicester. The authors are grateful for the support, in terms of equipment use, from the UK Collaboratorium for Research in Infrastructure and Cities (UKCRIC) and the Engineering and Physical Sciences Research Council (EPSRC) under capital grant number EP/P017169/1 (National Centre for Infrastructure Materials in Leeds).

Institutional Review Board Statement: Not applicable.

Informed Consent Statement: Not applicable.

Data Availability Statement: Available upon request from the corresponding author.

Acknowledgments: Martin Cockrill from Polytec UK is acknowledged for providing training on the use of laser Doppler vibrometer. Sumit S. Parale is acknowledged for providing assistance with the integration of OpenCV libraries. Vinay Patel from the School of Engineering and Advanced Microscopy Centre at the University of Leicester is acknowledged for providing images of lightreflective fiducial markers taken with a scanning electron microscope.

Conflicts of Interest: The authors declare no conflict of interest.

\section{References}

1. Feng, D.; Feng, M.Q. Computer vision for SHM of civil infrastructure: From dynamic response measurement to damage detection-A review. Eng. Struct. 2018, 156, 105-117. [CrossRef]

2. Xu, Y.; Brownjohn, J.M.W. Review of machine-vision based methodologies for displacement measurement in civil structures. J. Civ. Struct. Health Monit. 2018, 8, 91-110. [CrossRef]

3. Spencer, B.F.; Hoskere, V.; Narazaki, Y. Advances in computer vision-based civil infrastructure inspection and monitoring. Engineering 2019, 5, 199-222. [CrossRef]

4. Park, S.W.; Park, H.S.; Kim, J.H.; Adeli, H. 3D displacement measurement model for health monitoring of structures using a motion capture system. Meas. J. Int. Meas. Confed. 2015, 59, 352-362. [CrossRef]

5. Brownjohn, J.M.W.; Hester, D.; Xu, Y.; Bassitt, J.; Koo, K. Viability of optical tracking systems for monitoring deformations of a long span bridge. In Proceedings of the 6th European Conference on Structural Control, Sheffield, UK, 11-13 July 2016; European Association for the Control of Structures: Sheffield, UK, 2016.

6. Luo, L.; Feng, M.Q.; Wu, Z.Y. Robust vision sensor for multi-point displacement monitoring of bridges in the field. Eng. Struct. 2018, 163, 255-266. [CrossRef]

7. Patil, K.; Srivastava, V.; Baqersad, J. A multi-view optical technique to obtain mode shapes of structures. Meas. J. Int. Meas. Confed. 2018, 122, 358-367. [CrossRef]

8. Kromanis, R.; Xu, Y.; Lydon, D.; Martinez del Rincon, J.; Al-Habaibeh, A. Measuring structural deformations in the laboratory environment using smartphones. Front. Built Environ. 2019, 5. [CrossRef]

9. Zhao, X.; Ri, K.; Wang, N. Experimental verification for cable force estimation using handheld shooting of smartphones. J. Sens. 2017, 1-13. [CrossRef]

10. Feldbusch, A.; Sadegh-Azar, H.; Agne, P. Vibration analysis using mobile devices (smartphones or tablets). Procedia Eng. 2017, 199, 2790-2795. [CrossRef]

11. Lydon, D.; Lydon, M.; Taylor, S.; Del Rincon, J.M.; Hester, D.; Brownjohn, J. Development and field testing of a vision-based displacement system using a low cost wireless action camera. Mech. Syst. Signal Process. 2019, 121, 343-358. [CrossRef]

12. Fonseca, L.M.G.; Manjunath, B.S. Registration techniques for multisensor remotely sensed imagery. Photogramm. Eng. Remote Sens. 1996, 62, 1049-1056.

13. Xu, Y.; Brownjohn, J.; Kong, D. A non-contact vision-based system for multipoint displacement monitoring in a cable-stayed footbridge. Struct. Control Health Monit. 2018, 25, 1-23. [CrossRef]

14. Abdelbarr, M.; Chen, Y.L.; Jahanshahi, M.R.; Masri, S.F.; Shen, W.M.; Qidwai, U.A. 3D dynamic displacement-field measurement for structural health monitoring using inexpensive RGB-D based sensor. Smart Mater. Struct. 2017, 26. [CrossRef]

15. Fukuda, Y.; Feng, M.Q.; Shinozuka, M. Cost-effective vision-based system for monitoring dynamic response of civil engineering structures. Struct. Control Health Monit. 2010, 17, 918-936. [CrossRef]

16. Yang, Y.; Dorn, C.; Mancini, T.; Talken, Z.; Theiler, J.; Kenyon, G.; Farrar, C.; Mascareñas, D. Reference-free detection of minute, non-visible, damage using full-field, high-resolution mode shapes output-only identified from digital videos of structures. Struct. Health Monit. 2018, 17, 514-531. [CrossRef]

17. Dong, C.-Z.; Catbas, F.N. A review of computer vision-based structural health monitoring at local and global levels. Struct. Health Monit. 2020, 1475921720935585. [CrossRef]

18. Ewins, D.J. Modal Testing: Theory, Practice and Application, 2nd ed.; John Wiley \& Sons: Baldock, UK, 2000.

19. Guillaume, P.; Brussel, V.U.; De Troyer, T.; Brussel, V.U.; Devriendt, C.; Brussel, V.U.; De Sitter, G.; Brussel, V.U. OMAX-A combined experimental-operational modal analysis approach. In Proceedings of the International Conference on Noise and Vibration Engineering, ISMA 2006, Heverlee, Belgium, 18-20 September 2006; KTH: Stockholm, Sweden, 2006; pp. $2985-2996$. 
20. Reynders, E. System identification methods for (operational) modal analysis: Review and comparison. Arch. Comput. Methods Eng. 2012, 19, 51-124. [CrossRef]

21. Aenlle, M.; Juul, M.; Brincker, R. Modal mass and length of mode shapes in structural dynamics. Shock Vib. 2020, 2020, 8648769. [CrossRef]

22. Brincker, R.; Ventura, C. Introduction to Operational Modal Analysis; John Wiley \& Sons: Chichester, UK, 2015.

23. Brandt, A.; Berardengo, M.; Manzoni, S.; Cigada, A. Scaling of mode shapes from operational modal analysis using harmonic forces. J. Sound Vib. 2017, 407, 128-143. [CrossRef]

24. Oh, B.K.; Hwang, J.W.; Kim, Y.; Cho, T.; Park, H.S. Vision-based system identification technique for building structures using a motion capture system. J. Sound Vib. 2015, 356, 72-85. [CrossRef]

25. Reu, P.L.; Rohe, D.P.; Jacobs, L.D. Comparison of DIC and LDV for practical vibration and modal measurements. Mech. Syst. Signal Process. 2017, 86, 2-16. [CrossRef]

26. Yoon, H.; Elanwar, H.; Choi, H.; Golparvar-Fard, M.; Spencer, B.F. Target-free approach for vision-based structural system identification using consumer-grade cameras. Struct. Control Health Monit. 2016, 23, 1405-1416. [CrossRef]

27. Hoskere, V.; Park, J.W.; Yoon, H.; Spencer, B.F. Vision-based modal survey of civil infrastructure using unmanned aerial vehicles. J. Struct. Eng. 2019, 145, 1-14. [CrossRef]

28. Imetrum. Available online: https://www.imetrum.com/ (accessed on 20 April 2020).

29. Hester, D.; Brownjohn, J.; Bocian, M.; Xu, Y. Low cost bridge load test: Calculating bridge displacement from acceleration for load assessment calculations. Eng. Struct. 2017, 143, 358-374. [CrossRef]

30. Macdonald, J.H.G.; Dagless, E.L.; Thomas, B.T.; Taylor, C.A. Dynamic measurements of the Second Severn Crossing. Proc. Inst. Civ. Eng. Transp. 1997, 123, 241-248. [CrossRef]

31. OptiTrack. Available online: https:/ / optitrack.com/ (accessed on 20 April 2020).

32. Bradski, G.; Kaehler, A. Learning OpenCV: Computer Vision with the OpenCV Library; O'Reilly Media: Sebastopol, CA, USA, 2008.

33. Garrido-Jurado, S.; Muñoz-Salinas, R.; Madrid-Cuevas, F.J.; Marín-Jiménez, M.J. Automatic generation and detection of highly reliable fiducial markers under occlusion. Pattern Recognit. 2014, 47, 2280-2292. [CrossRef]

34. Popescu, D.C.; Cernaianu, M.O.; Ghenuche, P.; Dumitrache, I. An assessment on the accuracy of high precision 3D positioning using planar fiducial markers. In Proceedings of the 21st International Conference on System Theory, Control and Computing, ICSTCC 2017, Sinaia, Romania, 19-23 October 2017; pp. 471-476.

35. Zhang, Z. A flexible new technique for camera calibration. IEEE Trans. Pattern Anal. Mach. Intell. 2000, 22, 1330-1334. [CrossRef]

36. Feng, D.; Feng, M.Q.; Ozer, E.; Fukuda, Y. A vision-based sensor for noncontact structural displacement measurement. Sensors 2015, 15, 16557-16575. [CrossRef]

37. British Standards Institution. BS EN 12464-1:2002. Light and Lighting_Lighting of Work Places_Part 1: Indoor Work; BSI: London, UK, 2002.

38. Ye, X.W.; Ni, Y.Q.; Wai, T.T.; Wong, K.Y.; Zhang, X.M.; Xu, F. A vision-based system for dynamic displacement measurement of long-span bridges: Algorithm and verification. Smart Struct. Syst. 2013, 12, 363-379. [CrossRef]

39. Ozbek, M.; Rixen, D.J.; Erne, O.; Sanow, G. Feasibility of monitoring large wind turbines using photogrammetry. Energy 2010, 35, 4802-4811. [CrossRef]

40. Wahbeh, A.M.; Caffrey, J.P.; Masri, S.F. A vision-based approach for the direct measurement of displacements in vibrating systems. Smart Mater. Struct. 2003, 12, 785-794. [CrossRef]

41. Dong, C.Z.; Ye, X.W.; Liu, T. Non-contact structural vibration monitoring under varying environmental conditions. Vibroeng. Procedia 2015, 5, 217-222.

42. Olaszek, P. Investigation of the dynamic characteristic of bridge structures using a computer vision method. Meas. J. Int. Meas. Confed. 1999, 25, 227-236. [CrossRef]

43. Peeters, B.; Lowet, G.; Van der Auweraer, H.; Leuridan, J. A new procedure for modal parameter estimation. Sound Vib. 2004, $38,24-29$.

44. Peeters, B.; Van Der Auweraer, H.; Guillaume, P.; Leuridan, J. The PolyMAX frequency-domain method: A new standard for modal parameter estimation? Shock Vib. 2004, 11, 395-409. [CrossRef]

45. Guillaume, P.; Verboven, P.; Vanlanduit, S. A poly-reference implementation of the least-squares complex frequency-domain estimator. In Proceedings of the 21st International Modal Analysis Conference, Kissimmee, FL, USA, 3-6 February 2003.

46. Peeters, B.; Guillaume, P. The new PolyMAX Plus method: Confident modal parameter estimation even in very noisy cases. In Proceedings of the International Conference on Noise and Vibration Engineering, ISMA 2012/International Conference on Uncertainty in Structural Dynamics, USD 2012, Leuven, Belgium, 17-19 September 2012; pp. 2801-2814.

47. Smith, J.W. Vibration of Structures; Chapman \& Hall: London, UK, 1988.

48. Bocian, M.; Macdonald, J.H.G.; Burn, J.F. Probabilistic criteria for lateral dynamic stability of bridges under crowd loading. Comput. Struct. 2014, 136, 108-119. [CrossRef]

49. Brownjohn, J.M.W.; Bocian, M.; Hester, D.; Quattrone, A.; Hudson, W.; Moore, D.; Goh, S.; Lim, M.S. Footbridge system identification using wireless inertial measurement units for force and response measurements. J. Sound Vib. 2016, 384, 339-355. [CrossRef]

50. White, R.E.; Alexander, N.A.; Macdonald, J.H.G.; Bocian, M. Characterisation of crowd lateral dynamic forcing from full-scale measurements on the Clifton Suspension Bridge. Structures 2020, 24, 415-425. [CrossRef]

51. Nikitas, N.; Macdonald, J.H.G.; Jakobsen, J.B. Identification of flutter derivatives from full-scale ambient vibration measurements of the Clifton Suspension Bridge. Wind Struct. Int. J. 2011, 14, 221-238. [CrossRef] 
52. Bendat, J.S.; Piersol, A.G. Random Data: Analysis and Measurement Procedures, 4th ed.; John Wiley \& Sons: Hoboken, NJ, USA, 2010.

53. Allemang, R.J. The modal assurance criterion-Twenty years of use and abuse. Sound Vib. 2003, 37, 14-23.

54. Allemang, R.J.; Brown, D.L. A correlation coefficient for modal vector analysis. In Proceedings of the 1st International Modal Analysis Conference, Orlando, FL, USA, 8-10 November 1982; pp. 110-116.

55. Balmes, E. GARTEUR group on ground vibration testing. Results from the test of a single structure by 12 laboratories in Europe. In Proceedings of the 15th International Modal Analysis Conference, Orlando, FL, USA, 3-6 February 1997; Volume 2, pp. $1346-1352$.

56. Park, H.S.; Young Kim, J.; Gi Kim, J.; Woon Choi, S.; Kim, Y. A new position measurement system using a motion-capture camera for wind tunnel tests. Sensors 2013, 13, 12329-12344. [CrossRef] [PubMed]

57. Pan, B.; Qian, K.; Xie, H.; Asundi, A. Two-dimensional digital image correlation for in-plane displacement and strain measurement: A review. Meas. Sci. Technol. 2009, 20. [CrossRef]

58. Ribeiro, D.; Calçada, R.; Ferreira, J.; Martins, T. Non-contact measurement of the dynamic displacement of railway bridges using an advanced video-based system. Eng. Struct. 2014, 75, 164-180. [CrossRef]

59. Dai, K.; Ren, X.; Chen, Q.; Zhao, B. Case studies of LDV-aided dynamic testing. Appl. Mech. Mater. 2012, 226-228, 2066-2071. [CrossRef] 